\title{
MESONIC AND BINDING CONTRIBUTIONS TO THE EMC EFFECT IN A RELATIVISTIC MANY BODY APPROACH
}

\author{
E. Marco ${ }^{1}$, E. Oset ${ }^{1}$ and P. Fernández de Córdoba ${ }^{2}$ \\ ${ }^{1}$ Departamento de Física Teórica and IFIC, Centro Mixto Universidad de Valencia- \\ CSIC, 46100 Burjassot (Valencia), Spain \\ ${ }^{2}$ Departamento de Matemática Aplicada, Universidad Politécnica de Valencia
}

\begin{abstract}
We revise the conventional nuclear effects of Fermi motion, binding and pionic effects in deep inelastic lepton scattering using a relativistic formalism for an interacting Fermi sea and the local density approximation to translate results from nuclear matter to finite nuclei. In addition we also consider effects from $\rho$-meson renormalization in the nucleus. The use of nucleon Green's functions in terms of their spectral functions offers a precise way to account for Fermi motion and binding. On the other hand the use of many body Feynman diagrams in a relativistic framework allows one to avoid using prescriptions given in the past to introduce relativistic corrections in a non relativistic formalism.

We show that with realistic nucleon spectral functions and meson nucleus selfenergies one can get a reasonable description of the EMC effect for $x>0.15$, outside the shadowing region.
\end{abstract}




\section{Introduction}

The EMC effect [1] is probably one of the topics in the interplay of nuclear and particle physics which has attracted more attention. Pioneering work on Fermi motion and pionic effects [2, 3], 1] was followed by many different ideas like binding of the nucleons in the nucleus, multiquark cluster effects or $Q^{2}$ rescaling (see refs. 5, 6, 7] for reviews on the topic). Here we shall pay attention only to conventional nuclear degrees of freedom, mesons ( $\pi$ and $\rho$ ) and nucleons. One of the interesting ideas along these lines was the effect of the nuclear binding [8, 9, 10], which, with ups and downs, has come to be accepted as, largely or at least partly, responsible for the depletion of $R(x)=$ $2 F_{2 A}(x) / A F_{2 D}(x)$ in the region of the minimum.

Criticism on this latter works was raised in [11], where it was shown that the introduction of relativistic corrections in the usual nuclear nonrelativistic treatment of the binding effects resulted in a flux factor which reduced the conventional binding effects [11, 12]. This flux factor led to a different normalization of the spectral function which preserved the baryonic number calculated relativistically [11]. The argument of the normalization of the baryonic number is an important one and the idea has met with followers [12, 13, 14. However, it is a prescription on how to convert the nonrelativistic nuclear wave function into a relativistic spectral function [15] and the prescription is not shared by others [6]. Furthermore, these are not the only relativistic corrections as shown in 16, 17].

This issue justifies a work like the present one, where we construct from the beginning a relativistic nucleon spectral function and define everything within a field theoretical formalism which uses the nucleon propagators written in terms of this spectral function. The relativistic formalism is taken from the beginning and the baryonic number is naturally well normalized. Since all the nuclear information needed is contained in the nucleon spectral function, one does not need to use ordinary nuclear wave functions, which are anyway static (no spreading in the energy distribution, which is just concentrated in the single particle energies of the shell model) and hence one does not need any step to introduce relativistic effects into the nonrelativistic wave functions, as done in [11]. Furthermore, the use of non static spectral functions is important.

This was already seen in refs. [13, 15] which showed that the use of more realistic spectral functions accounting for nuclear correlations resulted in an enhancement of the binding effects. The reason is that, for a given average binding energy, the approach with a realistic spectral function leads to a larger kinetic and potential energy in absolute value than the shell model approach.

The other issue that we have revisited here is the pionic contribution. Large effects from the pionic cloud associated with the pion excess number in the nucleus were found in [2, 3, 7]. We have taken up the idea and recalculated these effects within the many body field theoretical approach using input which has been checked in a variety of nuclear reactions testing real and virtual pions: pionic reactions [18], muon capture [19], inclusive neutrino scattering [20] and 
photonuclear reactions 21. In addition we have also included corrections from the modification of $\rho$-meson cloud in the nucleus, in complete analogy with the pionic contribution.

Altogether we find that the use of the spectral function for the nucleons together with the mesonic effects can approximately account for the measured EMC effect.

The calculations are done using the spectral function for nucleons in nuclear matter, followed by the local density approximation. This was shown to be an excellent tool to deal with photonuclear reactions in the absence of shadowing effects [21]. Hence, a natural limit of our results is the region of shadowing, $x \leq$ 0.15 , where indeed there are discrepancies with the data, and other ingredients should be considered that we do not want to tackle [22, 23.

\section{Relativistic nucleon propagator in nuclear matter}

\subsection{Nonrelativistic nucleon propagator and spectral func- tions}

Let us recall first the nonrelativistic nucleon propagator for a noninteracting Fermi sea, which is given in momentum space by

$$
G\left(p^{0}, p\right)=\frac{1-n(\vec{p})}{p^{0}-\varepsilon(\vec{p})+i \epsilon}+\frac{n(\vec{p})}{p^{0}-\varepsilon(\vec{p})-i \epsilon}
$$

where $n(\vec{p})$ is the Fermi occupation number $n(\vec{p})=1$ for $|\vec{p}| \leq k_{F}, n(\vec{p})=0$ for $|\vec{p}|>k_{F}$ and $\varepsilon(\vec{p})$ is the nonrelativistic nucleon energy. Eq. (1) can be recast as

$$
G\left(p^{0}, p\right)=\frac{1}{p^{0}-\varepsilon(\vec{p})+i \epsilon}+2 \pi i n(\vec{p}) \delta\left(p^{0}-\varepsilon(\vec{p})\right)
$$

which separates the propagator into the free propagator and the medium correction.

For an interacting Fermi sea the nucleon propagator can be written in terms of its nonstatic selfenergy $\Sigma\left(p^{0}, p\right)$

$$
G\left(p^{0}, p\right)=\frac{1}{p^{0}-\varepsilon(\vec{p})-\Sigma\left(p^{0}, p\right)}
$$

which can be rewritten in terms of the spectral functions for holes and particles as [24]

$$
G\left(p^{0}, p\right)=\int_{-\infty}^{\mu} \frac{S_{h}(\omega, p)}{p^{0}-\omega-i \epsilon} d \omega+\int_{\mu}^{\infty} \frac{S_{p}(\omega, p)}{p^{0}-\omega+i \epsilon} d \omega
$$

with the following relationships 


$$
\begin{array}{r}
S_{h}\left(p^{0}, p\right)=\frac{1}{\pi} \frac{\operatorname{Im} \Sigma\left(p^{0}, p\right)}{\left[p^{0}-\varepsilon(\vec{p})-\operatorname{Re} \Sigma\left(p^{0}, p\right)\right]^{2}+\left[\operatorname{Im} \Sigma\left(p^{0}, p\right)\right]^{2}} \\
\text { for } p^{0} \leq \mu \\
S_{p}\left(p^{0}, p\right)=-\frac{1}{\pi} \frac{\operatorname{Im} \Sigma\left(p^{0}, p\right)}{\left[p^{0}-\varepsilon(\vec{p})-\operatorname{Re} \Sigma\left(p^{0}, p\right)\right]^{2}+\left[\operatorname{Im} \Sigma\left(p^{0}, p\right)\right]^{2}} \\
\text { for } p^{0}>\mu
\end{array}
$$

let us also recall that the momentum distribution of the nucleon in this interacting Fermi sea is given by

$$
\begin{gathered}
n_{I}(\vec{p})=\int_{-\infty}^{\mu} S_{h}(\omega, p) d \omega \\
1-n_{I}(\vec{p})=\int_{\mu}^{\infty} S_{p}(\omega, p) d \omega
\end{gathered}
$$

with the automatic sum rule

$$
\int_{-\infty}^{\mu} S_{h}(\omega, p) d \omega+\int_{\mu}^{\infty} S_{p}(\omega, p) d \omega=1
$$

In passing we also note that in physical reactions $n_{I}(\vec{p})$ does not factorize out in the physical cross sections because other factors dependent on $\omega$ and $\vec{p}$ appear simultaneously in the formulae, and restrictions due to energy and momentum conservation do not allow the infinite ranges in the $\omega$ integration required in eq. (6). Failure to realize that, and the naive substitution of $n(\vec{p})$ in eq. (1) by $n_{I}(\vec{p})$ of eq. (6), as sometimes done, leads to erroneous results which can be off by three orders of magnitude in some cases [25]. This gives us a warning that we should express all our magnitudes in terms of the spectral functions, not the momentum distributions.

\subsection{Relativistic nucleon propagator and spectral func- tions.}

The free relativistic nucleon propagator is given by

$$
\frac{\not p+M}{p^{2}-M^{2}+i \epsilon} \equiv \frac{M}{E(\vec{p})}\left\{\frac{\sum_{r} u_{r}(\vec{p}) \bar{u}_{r}(\vec{p})}{p^{0}-E(\vec{p})+i \epsilon}+\frac{\sum_{r} v_{r}(-\vec{p}) \bar{v}_{r}(-\vec{p})}{p^{0}+E(\vec{p})-i \epsilon}\right\}
$$

where we have separated in the second member the contribution from positive and negative energy states $[26] . M, E(\vec{p})$ in eq. (8) are the nucleon mass and the relativistic nucleon energy $\left(\vec{p}^{2}+M^{2}\right)^{1 / 2}$, and $u_{r}(\vec{p}), v_{r}(\vec{p})$ are the ordinary spinors which we take normalized as $\bar{u}_{r}(\vec{p}) u_{r}(\vec{p})=1$. We recall that $u_{r}(\vec{p})$ are functions of three momentum and they will be the only spinors which will appear in our framework.

The relativistic nucleon propagator for a noninterating Fermi sea is easily derived and, by analogy to eq. (2), can be written as 


$$
G\left(p^{0}, p\right)=\frac{\not p+M}{p^{2}-M^{2}+i \epsilon}+2 \pi i n(\vec{p})(\not p+M) \theta\left(p^{0}\right) \delta\left(p^{2}-M^{2}\right)
$$

which by means of the identity of eq. (8) can be recast as

$$
\begin{gathered}
G\left(p^{0}, p\right)=\frac{M}{E(\vec{p})}\left\{\sum_{r} u_{r}(\vec{p}) \bar{u}_{r}(\vec{p})\left[\frac{1-n(\vec{p})}{p^{0}-E(\vec{p})+i \epsilon}+\frac{n(\vec{p})}{p^{0}-E(\vec{p})-i \epsilon}\right]\right. \\
\left.+\frac{\sum_{r} v_{r}(-\vec{p}) \bar{v}_{r}(-\vec{p})}{p^{0}+E(\vec{p})-i \epsilon}\right\}
\end{gathered}
$$

Apart from the negative energy contribution, which will play no role in our problem, the only difference between eq. (10) and the nonrelativistic propagator of eq. (1) is the presence of the factor $M / E(\vec{p})$ and the projector over the space of positive energies $\sum_{r} u_{r}(\vec{p}) \bar{u}_{r}(\vec{p})$, which are both unity in the nonrelativistic approximation.

Now we proceed to construct the relativistic propagator in the interacting Fermi sea. We wish to sum the Dyson series for the diagrams shown in fig. 1, where although not shown, one would also have other sources of nucleon selfenergies. We will write them in terms of the operator $\Sigma\left(p^{0}, p\right)$. It will become clear later on that we only need the imaginary part of the nucleon propagator for the positive energy states, in which case we neglect from the beginning the negative energy states (their weight becomes negligible compared to the singular part of the positive energy propagator). Hence, for the purpose of the present problem, the nucleon propagator needed will be

$$
\begin{gathered}
G\left(p^{0}, p\right)=\frac{M}{E(\vec{p})} \sum_{r} u_{r}(\vec{p}) \bar{u}_{r}(\vec{p}) \frac{1}{p^{0}-E(\vec{p})}+ \\
\frac{M}{E(\vec{p})} \sum_{r} \frac{u_{r}(\vec{p}) \bar{u}_{r}(\vec{p})}{p^{0}-E(\vec{p})} \Sigma\left(p^{0}, p\right) \frac{M}{E(\vec{p})} \sum_{s} \frac{u_{s}(\vec{p}) \bar{u}_{s}(\vec{p})}{p^{0}-E(\vec{p})}+\ldots \\
=\frac{M}{E(\vec{p})} \sum_{r} \frac{u_{r}(\vec{p}) \bar{u}_{r}(\vec{p})}{p^{0}-E(\vec{p})-\bar{u}_{r}(\vec{p}) \Sigma\left(p^{0}, p\right) u_{r}(\vec{p}) \frac{M}{E(\vec{p})}}
\end{gathered}
$$

where we have used the fact that $\Sigma$ should be diagonal in spin for spin saturated matter which we only consider.

Comparison of eqs. (11) and (3) shows again the differences between the relativistic and nonrelativistic propagators.

The structure of eq. (11) allows one to define a spectral representation of the nucleon propagator by means of $S_{h}(\omega, p)$ and $S_{p}(\omega, p)$ as

$$
G\left(p^{0}, p\right)=\frac{M}{E(\vec{p})} \sum_{r} u_{r}(\vec{p}) \bar{u}_{r}(\vec{p})\left[\int_{-\infty}^{\mu} d \omega \frac{S_{h}(\omega, p)}{p^{0}-\omega-i \eta}\right.
$$




$$
\left.+\int_{\mu}^{\infty} d \omega \frac{S_{p}(\omega, p)}{p^{0}-\omega+i \eta}\right]
$$

with the relationships

$$
\begin{array}{r}
S_{h}\left(p^{0}, p\right)=\frac{1}{\pi} \frac{\frac{M}{E(\vec{p})} \operatorname{Im} \Sigma\left(p^{0}, p\right)}{}\left[\begin{array}{r}
\text { for } p^{0} \leq \mu \\
{\left[p^{0}-E(\vec{p})-\frac{M}{E(\vec{p})} \operatorname{Re} \Sigma\left(p^{0}, p\right)\right]^{2}+\left[\frac{M}{E(\vec{p})} \operatorname{Im} \Sigma\left(p^{0}, p\right)\right]^{2}}
\end{array}\right. \\
S_{p}\left(p^{0}, p\right)=-\frac{1}{\pi} \frac{\frac{M}{E(\vec{p})} \operatorname{Im} \Sigma\left(p^{0}, p\right)}{\left[p^{0}-E(\vec{p})-\frac{M}{E(\vec{p})} \operatorname{Re} \Sigma\left(p^{0}, p\right)\right]^{2}+\left[\frac{M}{E(\vec{p})} \operatorname{Im} \Sigma\left(p^{0}, p\right)\right]^{2}} \\
\text { for } p^{0}>\mu
\end{array}
$$

where for simplicity $\Sigma$ is now $\bar{u} \Sigma u$ which is independent of the spin. Eqs. (13) are now the generalizations of eqs. (5) using relativistic kinematics. Note that $S_{p}$ and $S_{h}$ defined in eq. (12) are not the nonrelativistic spectral functions normally used. Hence one should not expect the same normalization as in [11, 12, 14]. The normalization of $S_{h}$, which we will need, is easily obtained by imposing baryon number conservation, as done in [11]. For this purpose we evaluate the electromagnetic form factor at $q=0$. For the case of the nucleon (fig. 2a)) we have (assume all baryons have charge unity for normalization purposes),

$$
<N\left|B^{\mu}\right| N>\equiv \bar{u}(\vec{p}) \gamma^{\mu} u(\vec{p})=B \frac{p^{\mu}}{M} ; B=1, p^{\mu} \equiv(E(\vec{p}), \vec{p})
$$

For the case of nucleons in the medium we must evaluate the many body diagram of fig. $2 \mathrm{~b}$ ).

$$
<A\left|B^{\mu}\right| A>=(-) \int \frac{d^{4} p}{(2 \pi)^{4}} \operatorname{ViTr}\left[G\left(p^{0}, p\right) \gamma^{\mu}\right] e^{i p^{0} \eta}
$$

where $\exp \left(i p^{0} \eta\right)$, with $\eta \rightarrow 0^{+}$, is the convergence factor for loops appearing at the same time [24] and $\mathrm{V}$ the volume of our normalization box.

By means of eq. (12) we can see that the convergence factor limits the contribution to the hole spectral function and we get

$$
\begin{aligned}
<A\left|B^{\mu}\right| A>=V & \int \frac{d^{3} p}{(2 \pi)^{3}} \frac{M}{E(\vec{p})} \operatorname{Tr}\left[\sum_{r} u_{r}(\vec{p}) \bar{u}_{r}(\vec{p}) \gamma^{u}\right] \\
& \cdot \int_{-\infty}^{\mu} S_{h}(\omega, p) d \omega
\end{aligned}
$$




$$
\begin{gathered}
=V \int \frac{d^{3} p}{(2 \pi)^{3}} \frac{M}{E(\vec{p})} \operatorname{Tr}\left[\frac{(\not p+M)_{\text {on shell }}}{2 M} \gamma^{u}\right] \int_{-\infty}^{\mu} S_{h}(\omega, p) d \omega \\
=2 V \int \frac{d^{3} p}{(2 \pi)^{3}} \frac{M}{E(\vec{p})} \frac{p_{\text {on shell }}^{\mu}}{M} \int_{-\infty}^{\mu} S_{h}(\omega, p) d \omega \equiv B \frac{P_{A}^{\mu}}{M_{A}}
\end{gathered}
$$

where in the last step we have imposed that this matrix element gives the right current with B baryons, in analogy to eq. (14), and $P_{A}^{\mu}$ is the momentum of the nucleus. Note that $p_{\text {on shell }}^{\mu}$ appears in eq. (16) because the operator $(\not p+M)_{\text {on shell }}$ comes from $u_{r}(\vec{p}) \bar{u}_{r}(\vec{p})$ which depends only on $\vec{p}$ (it corresponds to free particles with $\left.p^{\mu} \equiv(E(\vec{p}), \vec{p})\right)$. Obviously eq. (16) must be evaluated in the rest frame of our Fermi sea where all magnitudes are defined. Only $\mu=0$ is then relevant and we obtain the desired normalization

$$
2 V \int \frac{d^{3} p}{(2 \pi)^{3}} \int_{-\infty}^{\mu} S_{h}(\omega, p) d \omega=B
$$

(Note that the factors $\frac{M}{E(\vec{p})}, \frac{p_{\text {on shell }}^{0}}{M}$ have cancelled in eq. (16)). The factor 2 is a spin factor. By a simple inspection of eq. (17) we can see that with the definition of the spectral functions in eq. (12) one can use eq. (6) to determine momentum distributions in both the nonrelativistic and relativistic cases.

In our formalism we do not have a box of constant density, but elements of volume $d^{3} r$ with local density $\rho_{p}(\vec{r}), \rho_{n}(\vec{r})$, the nuclear proton and neutron densities at the point $\vec{r}$. Hence our spectral functions for protons and neutrons are functions of the local Fermi momentum

$$
k_{F, p}(\vec{r})=\left[3 \pi^{2} \rho_{p}(\vec{r})\right]^{1 / 3} ; k_{F, n}(\vec{r})=\left[3 \pi^{2} \rho_{n}(\vec{r})\right]^{1 / 3}
$$

and then the equivalent normalization to eq. (17) is

$$
2 \int \frac{d^{3} p}{(2 \pi)^{3}} \int_{-\infty}^{\mu} S_{h}\left(\omega, p, k_{F p, n}(\vec{r})\right) d \omega=\rho_{p, n}(\vec{r})
$$

In practice we shall work with symmetric nuclear matter of density $\rho(\vec{r})$. Hence we have a unique Fermi momentum defined as $k_{F}(\vec{r})=\left[3 \pi^{2} \rho(r) / 2\right]^{1 / 3}$ and then one has

$$
4 \int \frac{d^{3} p}{(2 \pi)^{3}} \int_{-\infty}^{\mu} S_{h}\left(\omega, p, k_{F}(\vec{r})\right) d \omega=\rho(\vec{r})
$$

or equivalently

$$
\int d^{3} r \quad 4 \int \frac{d^{3} p}{(2 \pi)^{3}} \int_{-\infty}^{\mu} S_{h}\left(\omega, p, k_{F}(\vec{r})\right) d \omega=A
$$

with $A$ the mass number of each nucleus. The density $\rho(\vec{r})$ of each nucleus is taken from experiment in our case, and expressed in terms of a two Fermi parameter distribution for medium and heavy nuclei [27] and mod harmonic 
oscillator for light nuclei [27, 28]. Eq. (21) is fulfilled at the level of $2-3 \%$ in our case, in spite of the non trivial structure of the spectral function and the integrals involved. The small numerical deviation from the right normalization is taken care by dividing by the integral of eq. (21) instead of by $A$ in the evaluation of $R(x)$, since a similar integration weighed by the structure functions appears in the numerator, as we shall see. Although one could separate the contribution of protons and neutrons in the calculation, we have only applied the results to nuclei with $N=Z$, or very close, like ${ }^{56} F e$ and hence, we work with the symmetric nuclear matter version.

\section{Deep inelastic electron scattering from nu- clei}

Let us recall the basic ideas in deep inelastic scattering. Consider the $\left(e, e^{\prime}\right)$ process on a nucleon of fig. 3a). The invariant $T$ matrix for the process is

$$
-i T=i e \bar{u}_{e}\left(\overrightarrow{k^{\prime}}\right) \gamma^{\mu} u_{e}(\vec{k}) \frac{-i g_{\mu \nu}}{q^{2}}(-i e)<X\left|J^{\nu}\right| N>
$$

where $\left\langle X\left|J^{\nu}\right| N>\right.$ is the invariant matrix element of the hadronic current. The cross section for the process $e N \rightarrow e^{\prime} X$ is given in Mandl and Shaw normalization 29 by

$$
\begin{gathered}
\sigma=\frac{1}{v_{r e l}} \frac{2 m}{2 E_{e}(\vec{k})} \frac{2 M}{2 E(\vec{p})} \int \frac{d^{3} k^{\prime}}{(2 \pi)^{3}} \frac{2 m}{2 E_{e}\left(\vec{k}^{\prime}\right)} \\
\Pi_{i=1}^{N} \int \frac{d^{3} p_{i}^{\prime}}{(2 \pi)^{3}} \Pi_{l \epsilon f}\left(\frac{2 M_{l}^{\prime}}{2 E_{l}^{\prime}}\right) \Pi_{j \epsilon b}\left(\frac{1}{2 \omega_{j}^{\prime}}\right) \bar{\Sigma} \Sigma|T|^{2}(2 \pi)^{4} \\
\delta^{4}\left(p+k-k^{\prime}-\Sigma_{i=1}^{N} p_{i}^{\prime}\right)
\end{gathered}
$$

where $m$ is the electron mass, $f$ stands for fermions and $b$ for bosons in the

final state $\mathrm{X}$. The factor $\frac{2 M}{2 E(\vec{p})}$ becomes $\frac{1}{2 \omega(\vec{p})}$ if we study the cross section on a pion. The index $i$ is split in $l, j$ for fermions and bosons respectively.

In the nucleon rest frame one can then write the differential cross section, with $\Omega^{\prime}, E^{\prime}$ referring to the outgoing electron, as

$$
\frac{d^{2} \sigma}{d \Omega^{\prime} d E^{\prime}}=\frac{\alpha^{2}}{q^{4}} \frac{k^{\prime}}{k} L_{\mu \nu}^{\prime} W^{\prime \mu \nu}
$$

with $\alpha=e^{2} / 4 \pi$ and $L_{\mu \nu}^{\prime}$ the leptonic tensor

$$
L_{\mu \nu}^{\prime}=2 k_{\mu} k_{\nu}^{\prime}+2 k_{\mu}^{\prime} k_{\nu}+q^{2} g_{\mu \nu}
$$

and $W^{\prime \mu \nu}$ the hadronic tensor defined as

$$
W^{\prime \mu \nu}=\frac{1}{2 \pi} W^{\mu \nu}
$$


with

$$
\begin{aligned}
W^{\mu \nu} & =\bar{\Sigma}_{s_{p}} \Sigma_{X} \Sigma_{s_{i}} \Pi_{i=1}^{N} \int \frac{d^{3} p_{i}^{\prime}}{(2 \pi)^{3}} \Pi_{l \epsilon f}\left(\frac{2 M_{l}^{\prime}}{2 E_{l}^{\prime}}\right) \Pi_{j \epsilon b}\left(\frac{1}{2 \omega_{j}^{\prime}}\right) \\
& <X\left|J^{\mu}\right| H>^{*}<X\left|J^{\nu}\right| H>(2 \pi)^{4} \delta^{4}\left(p+q-\Sigma_{i=1}^{N} p_{i}^{\prime}\right)
\end{aligned}
$$

where $q$ is the momentum of the virtual photon, $s_{p}$ the spin of the nucleon and $s_{i}$ the spin of the fermions in $X$.

Lorentz covariance and gauge invariance allow one to write $W^{\prime \mu \nu}$ as 30

$$
W^{\prime \mu \nu}=\left(\frac{q^{\mu} q^{\nu}}{q^{2}}-g^{\mu \nu}\right) W_{1}+\left(p^{\mu}-\frac{p \cdot q}{q^{2}} q^{\mu}\right)\left(p^{\nu}-\frac{p \cdot q}{q^{2}} q^{\nu}\right) \frac{W_{2}}{M^{2}}
$$

where $W_{1}, W_{2}$ are the two structure functions of the nucleon and are functions of $q^{2}, p . q$.

Now we evaluate the cross section for $\left(e, e^{\prime}\right)$ on the nucleus. In order not to miss flux factors and be able to write everything in terms of propagators, we evaluate the electron selfenergy corresponding to the diagram in fig. $3 \mathrm{~b}$ ). We obtain

$$
\begin{gathered}
-i \Sigma(k)=\int \frac{d^{4} q}{(2 \pi)^{4}} \bar{u}_{e}(\vec{k}) i e \gamma^{\mu} i \frac{\not k^{\prime}+m}{k^{2}-m^{2}+i \epsilon} i e \gamma^{\nu} u_{e}(\vec{k}) \\
\frac{-i g_{\mu \rho}}{q^{2}}(-i) \Pi^{\rho \sigma}(q) \frac{-i g_{\sigma \nu}}{q^{2}}
\end{gathered}
$$

which for unpolarized electrons can be written as

$$
\Sigma(k)=i e^{2} \int \frac{d^{4} q}{(2 \pi)^{4}} \frac{1}{q^{4}} \frac{1}{2 m} L_{\mu \nu}^{\prime} \frac{1}{k^{\prime 2}-m^{2}+i \epsilon} \Pi^{\mu \nu}(q)
$$

with $\Pi^{\mu \nu}(q)$ the photon selfenergy.

In order to evaluate the cross section we need only $\operatorname{Im} \Sigma(k)$ which can be easily evaluated by means of Cutkosky rules 26.

$$
\begin{array}{ll}
\Sigma(k) & \rightarrow 2 i \operatorname{Im} \Sigma(k) \\
D\left(k^{\prime}\right) & \rightarrow 2 i \theta\left(k^{\prime 0}\right) \operatorname{Im} D\left(k^{\prime}\right) \text { (boson propagator) } \\
\Pi^{\mu \nu}(q) \rightarrow 2 i \theta\left(q^{0}\right) \operatorname{Im} \Pi^{\mu \nu}(q) \\
G(p) \rightarrow 2 i \theta\left(p^{0}\right) \operatorname{Im} G(p) \text { (fermion propagator) }
\end{array}
$$

with the result

$$
\operatorname{Im} \Sigma(k)=e^{2} \int \frac{d^{3} q}{(2 \pi)^{3}} \frac{1}{2 E_{e}(\vec{k}-\vec{q})} \theta\left(q^{0}\right) \operatorname{Im} \Pi^{\mu \nu}(q) \frac{1}{q^{4}} \frac{1}{2 m} L_{\mu \nu}^{\prime}
$$

with $q^{0}=k^{0}-E_{e}(\vec{k}-\vec{q})$. 
The cross section is readily evaluated from there. Inspection of eq. (11) for the relativistic fermion propagator tells us that the electron width is given by

$$
\Gamma(k)=-\frac{2 m}{E_{e}(\vec{k})} \operatorname{Im} \Sigma(k)
$$

by means of which the contribution to the cross section from an element of volume $d^{3} r$ in the rest frame of the nucleus is

$$
\begin{gathered}
d \sigma=\Gamma d t d S=\Gamma \frac{d t}{d l} d l d S=\frac{\Gamma}{v} d^{3} r= \\
=\Gamma \frac{E_{e}(\vec{k})}{k} d^{3} r=-\frac{2 m}{k} \operatorname{Im} \Sigma d^{3} r
\end{gathered}
$$

Hence we immediately write the $\left(e, e^{\prime}\right)$ cross section in the nucleus as

$$
\frac{d^{2} \sigma}{d \Omega^{\prime} d E^{\prime}}=-\frac{\alpha}{q^{4}} \frac{k^{\prime}}{k} \frac{1}{(2 \pi)^{2}} L_{\mu \nu}^{\prime} \int d^{3} r \operatorname{Im} \Pi^{\mu \nu}(q)
$$

with $q^{0}=k^{0}-E_{e}(\vec{k}-\vec{q})=k^{0}-k^{\prime 0}$, which is always positive in this experiment, so we drop the $\theta\left(q^{0}\right)$ function. Comparison of eq. (35) with eq. (24) used for nuclear targets tells as that

$$
W_{A}^{\prime \mu \nu}(q)=-\frac{1}{e^{2}} \frac{1}{\pi} \int d^{3} r \operatorname{Im} \Pi^{\mu \nu}(q)
$$

Next we evaluate $\Pi^{\mu \nu}(q)$ corresponding to the right hand side of the diagram of fig. 3b) using again the Feynman rules in terms of propagators. We have

$$
-i \Pi^{\mu \nu}(q)=(-) \int \frac{d^{4} p}{(2 \pi)^{4}} i G(p) \Sigma_{X} \Sigma_{s_{p}, s_{i}} \Pi_{i=1}^{N} \int \frac{d^{4} p_{i}^{\prime}}{(2 \pi)^{4}}
$$

$$
\Pi_{l} i G_{l}\left(p_{l}^{\prime}\right) \Pi_{j} i D_{j}\left(p_{j}^{\prime}\right)(-i)^{2} e^{2}<X\left|J^{\mu}\right| H><X\left|J^{\nu}\right| H>^{*}(2 \pi)^{4} \delta^{4}\left(q+p-\Sigma_{i=1}^{N} p_{i}^{\prime}\right)
$$

which by means of Cutkosky rules (31) and the use of free propagators, eq. (8), for the final states and the medium propagator, eq. (12), for $G(p)$, plus eq. (36), leads immediately to

$$
\begin{gathered}
W_{A}^{\prime \mu \nu}=\Sigma_{n, p} \int d^{3} r \int \frac{d^{3} p}{(2 \pi)^{3}} \frac{M}{E(\vec{p})} \int_{-\infty}^{\mu} S_{h}\left(p^{0}, p\right) d p^{0} \\
\frac{1}{2 \pi} \Sigma_{X} \Sigma_{s_{p}} \Sigma_{s_{i}} \Pi_{i=1}^{N} \int \frac{d^{3} p_{i}^{\prime}}{(2 \pi)^{3}} \Pi_{l \epsilon f}\left(\frac{2 M_{l}^{\prime}}{2 E_{l}^{\prime}}\right) \Pi_{j \epsilon b}\left(\frac{1}{2 \omega_{j}^{\prime}}\right) \\
<X\left|J^{\mu}\right| H><X\left|J^{\nu}\right| H>^{*}(2 \pi)^{4} \delta^{4}\left(q+p-\Sigma_{i=1}^{N} p_{i}^{\prime}\right)
\end{gathered}
$$


which by means of eq. (27) can be rewritten for an isospin symmetric nucleus as

$$
\begin{gathered}
W_{A}^{\prime \mu \nu}=4 \int d^{3} r \int \frac{d^{3} p}{(2 \pi)^{3}} \frac{M}{E(\vec{p})} \int_{-\infty}^{\mu} d p^{0} S_{h}\left(p^{0}, p\right) \\
W_{N}^{\prime \mu \nu}(p, q)
\end{gathered}
$$

with

$$
p \equiv\left(p^{0}, \vec{p}\right) ; W_{N}^{\prime \mu \nu}=\frac{1}{2}\left(W_{p}^{\prime \mu \nu}+W_{n}^{\prime \mu \nu}\right)
$$

Note that $W_{N}^{\prime \mu \nu}(p, q)$ appears with the off shell arguments of $p$, the bound nucleon.

In the steps from eq. (37) to (38) the spinors $u(\vec{p})$ are included in the matrix elements of the currents and we have considered that there is necessarily a fermion loop (hence the first minus sign in eq. (37)) with a free particle in the final state and the nucleon in the medium in the initial state. The corresponding energy integration in the loop (if we had used a Wick rotation explicitly instead of Cutkosky rules) necessarily picks up the hole part of the propagator of eq. (12), and at the same time relaxes the condition $\theta\left(p^{0}\right)$ of Cutkosky rules which does not appear for the hole part.

In eq. (39) there is an apparent lack of normalization, since assuming $W^{\prime \mu \nu}$ constant (which actually cannot be in practice) we would expect $W_{A}^{\prime \mu \nu}=$ $A W^{\prime \mu \nu}$. However given the normalization of the spectral function in eq. (21), this is not the case. In eq. (39) we get the extra factor $\frac{M}{E(\vec{p})}$ which does not appear in eq. (21). It is interesting to see the meaning of this factor in eq. (39). If we look at the formula of the $e N$ cross section in eq. (23), and by means of eqs. (25) and (27) we find

$$
\sigma=\frac{\alpha^{2} M}{v_{r e l} E_{e}(\vec{k}) E(\vec{p})} \int \frac{d^{3} k^{\prime}}{E_{e}\left(\vec{k}^{\prime}\right)} \frac{1}{q^{4}} L_{\mu \nu}^{\prime} W_{N}^{\prime \mu \nu}
$$

where $L_{\mu \nu}^{\prime} W^{\prime \mu \nu}$ is a Lorentz invariant and the content of the integral in eq. (40) also. For collinear frames of reference we also have

$$
v_{r e l} E_{e}(\vec{k}) E(\vec{p})=M k
$$

where $k$ is the electron momentum in the frame where the nucleon is at rest and hence

$$
\sigma=\frac{\alpha^{2}}{k} \int \frac{d^{3} k^{\prime}}{E_{e}\left(\vec{k}^{\prime}\right)} \frac{1}{q^{4}} L_{\mu \nu}^{\prime} W_{N}^{\prime \mu \nu}
$$

Now if we have a system of moving nucleons the cross section for scattering of the electron with the nucleus can not be obtained as a sum of individual cross sections, because the relative $e N$ flux is different for each nucleon. Instead, one has to sum the probabilities of collision per unit time for each nucleon and 
divide by a unique flux, the one relative to the $\mathrm{CM}$ of the nucleus. By taking for $v_{r e l}$ in eq. (40) the velocity of the electron with respect to the CM of the nucleus and suming over all the nucleons in eq. (40) we will be calculating the electron nucleus cross section. Hence we obtain in the rest frame of the nucleus

$$
\begin{gathered}
\sigma_{A}=\frac{\alpha^{2}}{k} \int \frac{d^{3} k^{\prime}}{E_{e}\left(\vec{k}^{\prime}\right)} \frac{1}{q^{4}} L_{\mu \nu}^{\prime} \sum_{\vec{p}} \frac{M}{E(\vec{p})} W_{N}^{\prime \mu \nu}(p, q) \\
=\frac{\alpha^{2}}{k} \int \frac{d^{3} k^{\prime}}{E_{e}\left(\vec{k}^{\prime}\right)} \frac{1}{q^{4}} L_{\mu \nu}^{\prime} 4 \int d^{3} r \int \frac{d^{3} p}{(2 \pi)^{3}} \frac{M}{E(\vec{p})} \int_{-\infty}^{\mu} d p^{0} S_{h}\left(p^{0}, p\right) W_{N}^{\prime \mu \nu}(p, q)
\end{gathered}
$$

where $k$ is the electron momentum in the nucleus rest frame. Since our nuclear cross section is given in terms of $W_{A}^{\prime \mu \nu}$ by (see eqs. (35), (36))

$$
\sigma_{A}=\frac{\alpha^{2}}{k} \int \frac{d^{3} k^{\prime}}{E_{e}\left(\vec{k}^{\prime}\right)} L_{\mu \nu}^{\prime} W_{A}^{\prime \mu \nu}
$$

then eq. (39) follows immediately.

The previous discussion has shown that the factor $\frac{M}{E(\vec{p})}$ is a factor appearing in the probability of reaction per unit time for each nucleon, and remains in the integral when we divide by a unique flux in order to obtain the nuclear cross section. It is thus a Lorentz contraction factor.

In the limit of small densities, when $M / E(\vec{p})=1$, eq. (39) with the consideration of eq. (21) would give $W_{A}^{\prime \mu \nu}=A W_{N}^{\prime \mu \nu}$ as it should be. Eq. (39) accounts for Fermi motion and binding and includes the relativistic Lorentz contraction factor $M / E(\vec{p})$ and the change of the arguments in $W^{\prime \mu \nu}(p, q)$.

Note in passing that the relativistic factor $m / E_{e}(\vec{k})$ of eq. $(33)$, which we extracted from the relativistic propagator of eq. (11), has been essential to provide the right normalization.

\section{Contribution from the pion cloud}

Let us first see the free pion structure function. The same formula eq. (24) is used for pions and this defines $W_{\pi}^{\prime \mu \nu}$. Given the normalization of the fields which we follow [29], the cross section of eq. (23) contains the factor $1 / 2 \omega(\vec{p})$ instead of $2 M / 2 E(\vec{p})$. Hence, this means that the definition $W_{\pi}^{\prime \mu \nu}$ is given by eq. (27) dividing the right hand side of the equation by $2 m_{\pi}$ (and obviously the average over the spin of the nucleon does not appear now for the pion case).

In order to derive the contribution from the virtual pions in the medium we evaluate again the electron selfenergy related to the diagram of fig. 4 . We can save all the steps given before simply by noting the differences in the two cases: 
i) The bound nucleon propagator is substituted by a pion propagator. From the use of Cutkosky rules we must change

$$
-2 \pi \frac{M}{E(\vec{p})} \int_{-\infty}^{\mu} d \omega S_{h}(\omega, p) \delta\left(p^{0}-\omega\right)
$$

by

$$
2 \theta\left(p^{0}\right) \operatorname{Im} D(p)
$$

with $D(p)$ the pion propagator (in the medium).

ii) One must take into account that $W_{\pi}^{\prime \mu \nu}$ is given by eq. (27) divided by $2 m_{\pi}$.

iii) The sum over spins of the bound nucleon in eq. (37) does not appear now for the case of the pion.

iv) There are three charged states of pions.

With only these four rules we can already write

$$
W_{A, \pi}^{\prime \mu \nu}=3 \int d^{3} r \int \frac{d^{4} p}{(2 \pi)^{4}} \theta\left(p^{0}\right)(-2) \operatorname{Im} D(p) 2 m_{\pi} W_{\pi}^{\prime \mu \nu}(p, q)
$$

Now there are two obvious subtractions to eq. (46). First one should subtract the contribution from a free pion, which has nothing to do with medium effects. However, this is zero because one electron can not decay into another electron, one pion and X. Then assuming the pion is dressed in the medium by exciting $p h$ and $\Delta h$ as we shall do, one is left with the contributions shown in fig. 5 .

Now there is no problem to get a contribution since $\operatorname{Im} D(p)$ gets strength from $p h$ excitation. The physical channel would correspond to $e \rightarrow e^{\prime}+X+p h$, or equivalently $e N \rightarrow e^{\prime} N^{\prime} X$, which is now allowed. The physical channels are easily visualized by cutting the intermediate states in the diagramas with a horizontal line and placing on shell the particles cut by the line. This is actually the essence of Cutkosky rules to obtain the imaginary part of the selfenergy of a diagram.

The former discussion also tells us that part of what we are calculating is already contained in the nucleon structure function. This is because we are also calculating the contribution from the pions contained in a free nucleon. This has to be subtracted. This is easily done by substituting in eq. (46)

$$
\operatorname{Im} D(p) \rightarrow \delta \operatorname{Im} D(p) \equiv \operatorname{Im} D(p)-\left.\rho \frac{\partial \operatorname{Im} D(p)}{\partial \rho}\right|_{\rho=0}
$$

since we substract $A$ times the contribution from the pion cloud to the structure function of the free nucleon. In technical words, we can say that we are only considering terms with at least two $p h$ or $1 p h 1 \Delta h$ (the most important terms) etc. in fig. 5 (up to Pauli blocking corrections in the $p h$ Lindhard function, which are automatically included by the procedure of eq. (47)).

Hence the genuine pionic contribution is given by 


$$
W_{A, \pi}^{\prime \mu \nu}=3 \int d^{3} r \int \frac{d^{4} p}{(2 \pi)^{4}} \theta\left(p^{0}\right)(-2) \delta \operatorname{Im} D(p) 2 m_{\pi} W_{\pi}^{\prime \mu \nu}(p, q)
$$

Eqn. (39) and (48) are the basic equations which provide the nucleonic and pionic contributions.

In passing we can mention that the distribution of the excess number of pions, per unit volume in the nucleus, $\delta N_{\pi}(p)$, which contains the averages of $<a_{p}^{+} a_{p}>,<a_{p}^{+} a_{-p}^{+}>$and $<a_{p} a_{-p}>$, is given by [31]

$$
\frac{\delta N_{\pi}(\vec{p})}{2 \omega(\vec{p})}=-3 \int_{0}^{\infty} \frac{d p^{0}}{2 \pi} \delta \operatorname{Im} D(p)
$$

such that in the case of a structure function $W_{\pi}^{\prime \mu \nu}(p, q)$ independent of $p^{0}$, eq. (48) could be considered as a weighed integral of the pion structure function over the pion excess distribution in the nucleus. However, the strong dependence of $W_{\pi}^{\prime \mu \nu}(p, q)$ on $p^{0}$ (imposed by energy and momentum conservation) does not allow that factorization, and hence a relationship of the pion excess number with the pionic contribution to the structure function cannot be established. The apparent extra factor 2 which we obtain in this counting (apart from the Lorentz contraction factor, $m_{\pi} / \omega(\vec{p})$ ) is obtained because one is automatically accounting for the imaginary part of the Compton $\gamma \pi$ amplitude which is crossing symmetric and contains the two diagrams of fig. 6, while the pion structure function for on shell pions contains only the imaginary part of the diagram 6a). (see refs. [31] and [32] for an elaborate discussion of these issues in the problem of the pion cloud contribution to $K^{+}$nucleus scattering). It is worth noting that there is no overlap between the Feynman diagrams accounted for in the pionic contribution, fig 5 , and those of the nucleonic contribution with Fermi motion and binding, which come from selfenergy insertions in the nucleon line of fig. 3b). Hence, these contributions to the nuclear structure function are independent.

\section{The Bjorken limit}

We have evaluated the contribution of nucleons and pions to the hadron structure function of the nucleus. We now proceed to write these expressions in terms of the Bjorken structure functions [33].

For nucleons (and similarly for pions or the nucleus) one introduces the Bjorken variables

$$
x=\frac{-q^{2}}{2 p q} ; \nu=\frac{p \cdot q}{M} ; Q^{2}=-q^{2}
$$

and for large values of $q^{0}$ and $Q^{2}$ simultaneously and fixed $x$ one has the Bjorken scaling

$$
\nu W_{2}\left(x, Q^{2}\right) \equiv F_{2}(x)
$$




$$
M W_{1}\left(x, Q^{2}\right) \equiv F_{1}(x)
$$

and the Callan-Gross relation

$$
2 x F_{1}(x)=F_{2}(x)
$$

up to some, $Q C D$ corrections in $\ln Q^{2}$. Since the same $Q^{2}$ will be chosen for the nucleus and the nucleon and we perfom ratios of structure functions we shall not worry about this dependence here.

In view of the relations (51), (52) the most practical way to proceed is to work with transverse components of $W^{\prime \mu \nu}$. For this purpose assume $\vec{q}$ along the $z$ direction, as usually done in the study of the $e, e^{\prime}$ reaction, and evaluate $W^{\prime x x}$. We find from eq. (28)

$$
\begin{aligned}
W^{\prime x x}=W_{1}+\frac{\left(p_{x}\right)^{2}}{M^{2}} W_{2} & \equiv \frac{F_{1}(x)}{M}+\frac{\left(p_{x}\right)^{2}}{M^{2}} \frac{F_{2}(x)}{\nu} \\
& =\frac{F_{1}(x)}{M} \text { in the Bjorken limit }
\end{aligned}
$$

this component has the virtue that the coefficient of $W_{1}$ is independent of $p$ and hence is the same for on shell or off shell nucleons, or pions, or the nucleus. Hence we can write

$$
\begin{gathered}
\frac{F_{1 A, N}\left(x_{A}\right)}{M_{A}}=4 \int d^{3} r \int \frac{d^{3} p}{(2 \pi)^{3}} \frac{M}{E(\vec{p})} \int_{-\infty}^{\mu} d p^{0} S_{h}\left(p^{0}, p\right) \frac{F_{1 N}\left(x_{N}\right)}{M} \\
\frac{F_{1 A, \pi}\left(x_{A}\right)}{M_{A}}=3 \int d^{3} r \int \frac{d^{4} p}{(2 \pi)^{4}} \theta\left(p^{0}\right)(-2) \delta \operatorname{Im} D(p) 2 m_{\pi} \frac{F_{1 \pi}\left(x_{\pi}\right)}{m_{\pi}} \\
x_{A}=-q^{2} / 2 M_{A} q^{0} \equiv \frac{x}{A} \quad, \quad \text { with } x=-q^{2} / 2 M q^{0} \\
x_{N}=-q^{2} / 2 p q \quad ; \quad x_{\pi}=-q^{2} /-2 p q
\end{gathered}
$$

where the extra minus sign in $x_{\pi}$ is because of the direction of $p$ in fig. 4 .

Here $F_{1 N}(x)=\left(F_{1 p}(x)+F_{1 n}(x)\right) / 2$ as implicit in eq. (39).

We still have to exert some caution since $0<x_{N}<1$. On the other hand $x_{\pi}<1$ but $x_{\pi}>x$ because in our scheme the emerging particle from the coupling of the pion to a nucleon of the nucleus is on shell when we excite $p h, \Delta h$ with the pion and take the imaginary part of $D(p)$ [3].

Since usually one compares ratios of the $F_{2}$ structure functions, this is easily accomplished by making use of the Callan-Gross relation (52) and we find 


$$
F_{2 A, N}\left(x_{A}\right)=4 \int d^{3} r \int \frac{d^{3} p}{(2 \pi)^{3}} \frac{M}{E(\vec{p})} \int_{-\infty}^{\mu} d p^{0} S_{h}\left(p^{0}, p\right) \frac{x}{x_{N}} F_{2 N}\left(x_{N}\right) \theta\left(x_{N}\right) \theta\left(1-x_{N}\right)
$$

$$
F_{2 A, \pi}\left(x_{A}\right)=-6 \int d^{3} r \int \frac{d^{4} p}{(2 \pi)^{4}} \theta\left(p^{0}\right) \delta \operatorname{Im} D(p) \frac{x}{x_{\pi}} 2 M F_{2 \pi}\left(x_{\pi}\right) \theta\left(x_{\pi}-x\right) \theta\left(1-x_{\pi}\right)
$$

where we have again $F_{2 N}=\left(F_{2 p}+F_{2 n}\right) / 2$ as implicit in eq. (39).

In the Bjorken limit the evaluation of eq. (55) does not require the knowledge of the variable $q$ since

$$
\frac{x}{x_{N}} \rightarrow \frac{p^{0}-p^{3}}{M} \quad ; \quad \frac{x}{x_{\pi}}=\frac{-p^{0}+p^{3}}{M}
$$

but it is implicit in the structure functions which are taken at a certain $Q^{2}$.

When reaching this point it is worth considering also the contribution of the $\rho$ meson cloud. Both the pion and the $\rho$ meson couple to nucleons and delta with derivative couplings, which give rise to relatively large meson selfenergies in the range of momenta which contributes to the structure functions. Furthermore, as found already in ref. [31], the pion cloud contribution comes mostly from the combined $p h$ and $\Delta h$ excitation, with the $p h$ on shell (in $\delta \operatorname{Im} D(p))$, and one finds a negligible contribution of two $p h$ excitations. Only $\pi$ and $\rho$ can excite the $\Delta h$ components and this makes these two mesons special when looking at the mesonic contribution to the nuclear structure function. In addition there is an interplay between $\pi$ and $\rho$ exchange. Indeed, the $\rho$ meson, through nuclear correlations, contributes both to the longitudinal and transverse parts of the spin-isospin $p h$ and $\Delta h$ interaction and it is an important element contributing to the Landau-Migdal $g^{\prime}$ parameter in a microscopic derivation of this interaction [34]. The value of this parameter governs to some extend the pionic (and $\rho$-meson) contribution to the structure function [3].

The contribution of the $\rho$-meson cloud to the structure function is given, by analogy to eq. (56) by

$$
\begin{gathered}
F_{2 A, \rho}\left(x_{A}\right)=-12 \int d^{3} r \int \frac{d^{4} p}{(2 \pi)^{4}} \theta\left(p^{0}\right) \delta \operatorname{Im}_{\rho}(p) \frac{x}{x_{\rho}} 2 M F_{2 \rho}\left(x_{\rho}\right) \\
\theta\left(x_{\rho}-x\right) \theta\left(1-x_{\rho}\right)
\end{gathered}
$$

where $D_{\rho}(p)$ is now the $\rho$-meson propagator and $F_{2 \rho}\left(x_{\rho}\right)$ is the $\rho$-meson structure function, which we take equal to the one of the pion following refs. [35, 36]. In addition $x_{\rho}$ is also given, in analogy to eq. (57), by

$$
\frac{x}{x_{\rho}}=\frac{-p^{0}+p^{3}}{M}
$$


Eq. (58) contains an extra factor of two compared to the pionic contribution of eq. (56). This is because of the two transverse polarizations of the $\rho$-meson and the fact that the coupling of the $\rho$ to nucleons and deltas which we consider, following ref. [34] is only of transverse nature $((\vec{\sigma} \times \vec{p}) \vec{\epsilon}$ for nucleons and $(\vec{S}+\times \vec{p}) \vec{\epsilon}$ for deltas, with $\vec{\epsilon}$ the polarization vector of the $\rho$ meson and $\vec{S}^{+}$the spin transition operator from spin $1 / 2$ to $3 / 2$ ).

The expression of $F_{1 A, N}\left(x_{A}\right)$ in eq. (54) shows the same lack of normalization discussed in connection with eq. (39), since assuming $F_{1 N}\left(x_{N}\right)$ constant (which is not the case) $F_{1 A, N}$ is not $A$ times $F_{1 N}$, due to the Lorentz contraction factor $\frac{M}{E(\vec{p})}$. The same can be said about eq. (55) in general. However, eq. (55), shows a particular normalization property. Indeed, if we take an ensemble of nucleons on shell $\left(p^{0}=E(\vec{p})\right)$ and $x=0$ (and hence $x_{N}=0$, by virtue of eq. (57)), then the Lorentz contraction factor $\frac{M}{E(\vec{p}))}$ cancells in average the dynamical factor $\frac{x}{x_{N}}$ and we get $F_{2 A, N}(0)=A F_{2 N}(0)$ for on shell nucleons. This cancellation, however, will not show up when the nucleons are off shell since in our formalism we still obtain the factor $\frac{M}{E(\vec{p})}$ but the dynamical factor $\frac{x}{x_{N}}$ will now be different.

In this discussion we are implicitly assuming that eqs. (54) and (55) stand as they are for the case of off shell nucleons simply by taking for $x_{N}$ the expression of eq. (57) using the nucleon off shell variables. This is certainly the easiest form of the analytical continuation in the off shell regime, although there have been other prescriptions in the Literature [37, 38, 39].

We would like to justify our assumption. Indeed, in our frawework we use nucleon propagators which are based on the free spinors (eq. (12)). Hence in eq. (38) the matrix elements would be defined in terms of free spinors, the final particles are free particles and the off shell dependence appears only in the $\delta($ ) function. Obviously one can not look in detail at all channels implicit in eq. (38). However, one can use the same philosophy in the parton model which is used to find out the scaling of the structure functions. We follow here the steps of ref. 33. and assume that partons carry a fraction $x_{q}$ of the nucleon momentum $p$ and its mass $M$, that the electron parton amplitude is given by the on shell expression and that the outgoing parton is a free one, but the $\delta()$ function appears with the off shell variables.

One finds then

$$
\begin{aligned}
W_{1}=W^{\prime} x x & =\sum_{i} \int d x_{q} f_{i}\left(x_{q}\right) e_{i}^{2} \frac{1}{2}\left(-q^{2}\right) \frac{1}{x_{q} M} \delta\left(q^{2}+2 p q x_{q}\right) \\
& =\sum_{i} e_{i}^{2} f_{i}\left(x_{N}\right) \frac{1}{2 M}=\frac{F_{1}\left(x_{N}\right)}{M} ; x_{N}=\frac{-q^{2}}{2 p q}
\end{aligned}
$$

where $F_{1}$ appears whith argument $x_{N}$ which is defined in terms of the off shell variables. Similarly one obtains

$$
\frac{p q}{M} W_{2}=\sum_{i} e_{i}^{2} f_{i}\left(x_{N}\right) x_{N}=F_{2}\left(x_{N}\right)
$$


whith $p . q$, and $x_{N}$ defined in terms of the off shell variables.

We would like to note here that our finding $F_{2 A}(0) \neq A F_{2 N}(0)$, or equivalently $R(0) \neq 1$, where $R(x)=F_{2 A}(x) / A F_{2 N}(x)$, is not so unconventional. Indeed, in ref. [16] the authors also find this property and they literally quote "Note that a feature of the results is that $R(0) \neq 1 \ldots$ It does not reflect any violation of baryon conservation, which is ensured by the normalization condition", in our case eq. (21).

The issue of the normalization still stirs much controversy. We have devoted many thoughts to it throughout this paper looking at it from some points of view not discussed before. We see that for an ensemble of uncorrelated nucleons $F_{2 A, N}(0)=A F_{2 N}(0)$ but as soon as interactions are accounted for this normalization is lost. A similar thing would happen should we evaluate the structure function $F_{3 A}$, which appears in neutrino scattering. This structure function when integrated over $x$ is normalized to 3 for the nucleon if one assumes models with only three valence quarks, or to $3 A$ for the nucleus. Once again this normalization would be lost if QCD interaction corrections are accounted for 40] and equivalently if $\mathrm{NN}$ interactions are included in the nuclear case, and experimentally this is the case 40. Also, experimentally $F_{2 A, N}(0) \neq A F_{2 N}(0)$. Clear as the question looks to us, we are aware that this point of view is not universally accepted. Further thoughts and discussions on the issue should be welcome which would help settle the question in a way acceptable to all.

Eqs. $(55,56,58)$ are the final equations which we use in the analysis.

For the nucleon and pion structure functions $F_{2 N}(x), F_{2 \pi}(x)$ we take the experimental values of refs. [41, 42].

\section{The meson propagators}

The pion propagator in the medium is given by

$$
D(p)=\left[p^{02}-\vec{p}^{2}-m_{\pi}^{2}-\Pi_{\pi}\left(p^{0}, p\right)\right]^{-1}
$$

with $\Pi_{\pi}$ the pion selfenergy. We consider the contribution of the $p h$ and $\Delta h$ excitations to the pion selfenergy in connection with the Landau-Migdal correction in the pionic channel, as well as off shell pion nucleon form factors.

Since for $\delta \operatorname{Im} D$ we need $D-D_{0}$, it is practical to write

$$
D(p)-D_{0}(p)=D_{0}^{2}(p) \frac{\frac{f^{2}}{m_{\pi^{2}}} F^{2}(p) \vec{p}^{2} \Pi^{*}(p)}{1-\frac{f^{2}}{m_{\pi}^{2}} V_{L}^{\prime}(p) \Pi^{*}(p)}
$$

where $F(p)$ is the $\pi N N$ form factor, which we take of the monopole type

$$
F(p)=\frac{\Lambda^{2}-m_{\pi^{2}}}{\Lambda^{2}-p^{2}}
$$


with $\Lambda=1300 \mathrm{MeV}$ 43 and $f^{2} / 4 \pi=0.08 . V_{L}^{\prime}(p)$ is the longitudinal part of the spin-isospin interaction and $\Pi^{*}(p)$ is the irreducible pion selfenergy, which contains all selfenergy diagrams which are not connected by $V_{L}^{\prime}(p)$.

For the $\rho$-meson we can write

$$
D_{\rho}(p)-D_{0 \rho}(p)=D_{0 \rho}^{2}(p) \frac{\frac{f^{2}}{m_{\pi^{2}}} C_{\rho} F_{\rho}^{2}(p) \vec{p}^{2} \Pi^{*}(p)}{1-\frac{f^{2}}{m_{\pi}^{2}} V_{T}^{\prime}(p) \Pi^{*}(p)}
$$

with $V_{T}^{\prime}(p)$ the transverse part of the spin-isospin interaction, $C_{\rho}=3.94$ 43. and $F_{\rho}(p)$ the $\rho N N$ form factor given by

$$
F_{\rho}(p)=\frac{\Lambda_{\rho}^{2}-m_{\rho}^{2}}{\Lambda_{\rho}^{2}-p^{2}}
$$

and $\Lambda_{\rho}=1400 \mathrm{MeV}$ 43.

For $V_{L}^{\prime}(p), V_{T}^{\prime}(p)$ we take the expressions which are derived from a model with $\pi$ and $\rho$ exchange in the presence of short range nuclear correlations [44, which are given by

$$
\begin{gathered}
V_{L}^{\prime}(p)=\vec{p}^{2} D_{0}(p) F^{2}(p)-\vec{p}^{2} \tilde{D}_{0}(p) \tilde{F}^{2}(p) \\
-\frac{1}{3} q_{c}^{2} \tilde{D}_{0}(p) \tilde{F}^{2}(p)-\frac{2}{3} q_{c}^{2} \tilde{D}_{0 \rho}(p) \tilde{F}_{\rho}^{2}(p) C_{\rho} \\
V_{T}^{\prime}(p)=\vec{p}^{2} D_{0 \rho}(p) F_{\rho}^{2}(p) C_{\rho}-\frac{1}{3} q_{c}^{2} \tilde{D}_{0}(p) \tilde{F}^{2}(p) \\
-\left(\vec{p}^{2}+\frac{2}{3} q_{c}^{2}\right) \tilde{D}_{0 \rho}(p) \tilde{F}_{\rho}^{2}(p) C_{\rho}
\end{gathered}
$$

Here $q_{c} \simeq 780 \mathrm{MeV}$ is the inverse of a typical correlation distance and $\tilde{D}(p), \tilde{F}(p), \tilde{D}_{\rho}(p), \tilde{F}_{\rho}(p)$ are the corresponding propagators and form factors substituting $\vec{p}^{2}$ by $\vec{p}^{2}+q_{c}^{2}$. The irreducible selfenergy $\Pi^{*}(p)$ is in our case the sum of the Lindhard functions $U_{N}(p), U_{\Delta}(p)$ for $p h$ and $\Delta h$ excitation with the normalization and analytical expressions of the appendix of ref. 45. It is interesting to note that for the values of $p^{0}, p$ which contribute most to the structure function, both $V_{L}^{\prime}(p)$ and $V_{T}^{\prime}(p)$ are negative and this leads to positive values of $F_{2 A, \pi}$ and $F_{2 A, \rho}$.

It was also found in 31 that in order to evaluate the contribution of the pion cloud by using the pion propagator, as done here, it is important that it satisfies the sum rule

$$
-\int_{0}^{\infty} \frac{d p^{0}}{\pi} 2 p^{0} \operatorname{Im} D\left(p^{0}, p\right)=1
$$

which expresses the equal time commutation relation of the pion fields. Our model satisfies this equation at the level of one per thousand. 


\section{The nucleon spectral function}

Section 2.2 has established the framework for the relativistic nucleon propagator which we need here. The only input needed is the nucleon selfenergy. We take it from the work of ref. [46]. This is a semiphenomenological, quite succesful approach, which uses as input the $N N$ cross section and the spin-isospin effective interaction. This allows one to evaluate $\operatorname{Im} \Sigma$, which is remarkably close to $\operatorname{Im} \Sigma$ of the elaborate many body calculations of ref. [47, 48]. The real part is evaluated by means of a dispersion relation, and the Fock term from the pionic contribution is also included. Only pieces of the Hartree type, which should be independent of the momentum, are missing for which one needs more information. Hence, up to an unknown momentum independent piece in the selfenergy the rest of the nucleon properties in the medium can be calculated, like effective masses, spectral functions, etc, which are also in good agreement with sofisticated many body calculations [49, 50]. Actually, what might appear a drawback is now a welcome feature because since the proper binding energy is an important ingredient in the $E M C$ effect, we also include phenomenologically a function $C(\rho)$ in the nucleon selfenergy and demand that the binding energy per nucleon be the experimental one for each nucleus. Then the model is complete, realistic and technically much simpler to handle than the sofisticated many body calculations [47, 49, 50].

With this improvement, momentum distributions and average binding energies are also in good agreement with other infinite nuclear matter [51] and finite nuclei calculations 52 .

A small inconvenience appears because the selfenergy of ref. [46] is evaluated non relativistically. However we have checked that a proper calculation including relativistic factors of the type $M / E(\vec{p})$ in the nucleon propagators in the integrals over the loops which appear in the evaluation of $\operatorname{Im} \Sigma$ in [46], would only introduce corrections in $\operatorname{Im} \Sigma$ below the level of $10 \%$. Second, we have changed $\operatorname{Im} \Sigma$ by $10 \%$ and found that the ratio $R(x)$ changes only at the level of $1 \%$. Thus we take the values for $\Sigma$ from ref. 446 and use them in the relativistic propagators of section 2.2. For the average kinetic and total nucleon energy we have

$$
\begin{aligned}
& <T>=\frac{4}{A} \int d^{3} r \int \frac{d^{3} p}{(2 \pi)^{3}}(E(\vec{p})-M) \int_{-\infty}^{\mu} S_{h}\left(p^{0}, p\right) d p^{0} \\
& <E>=\frac{4}{A} \int d^{3} r \int \frac{d^{3} p}{(2 \pi)^{3}} \int_{-\infty}^{\mu} S_{h}\left(p^{0}, p\right) p^{0} d p^{0}
\end{aligned}
$$

and the binding energy per nucleon is then given by the sum rule 53.

$$
\left|E_{A}\right|=-\frac{1}{2}\left(<E-M>+\frac{A-1}{A-2}<T>\right)
$$

which is also used in [54, 55] in connection with the study of the $E M C$ effect. We take experimental numbers for each nucleus for $\left|E_{A}\right|$ and adjust the func- 
tion $C(\rho)$ to fit $\left|E_{A}\right|$. We take $C(\rho)$ linear in the density, $C \rho(r)$. This quantity provides around $30 \mathrm{MeV}$ repulsion at $\rho=\rho_{0}$ in most of the nuclei. Detailed values for $\langle T\rangle,\langle E\rangle$ and $E_{A}$ can be seen in Table I.

As noted in refs. [13, 54, 55], the use of nucleon propagators in terms of non static spectral functions leads to bigger values of the average kinetic energy and $|\langle E-M\rangle|$ than the shell model of the nucleus and as a consequence to reduced values of $R(x)$ (for $x<0.7$ ). We can see this here also by taking the uncorrelated Fermi sea and adding a function $D \rho(r)$ to the ordinary ThomasFermi potential $V_{T F}(r)=-k_{F}(r)^{2} / 2 M$ such as to get the same binding energy via eq. (71). We can make use of the same formalism by simply considering that

$$
\begin{aligned}
S_{h}^{U F S}\left(p^{0}, p\right) & =n(\vec{p}) \delta\left(p^{0}-E(\vec{p})-\Sigma\right) \\
\Sigma(r) & =V_{T F}(r)+D \rho(r)
\end{aligned}
$$

Eq. (72) associates one energy to a given momentum, (the essence of the shell model in infinite nuclear matter) while the spectral function has a peak around the quasiparticle energy and then spreads out at larger values of $\vec{p}$ for a given energy. This results in a larger value of the average kinetic energy.

In Table I we show the results for different nuclei and compare them with those of ref. 54. As one can see, the results that we obtain with the uncorrelated Fermi sea and the spectral functions are remarkably close respectively to those of the Hartree Fock and spectral function used in [54.

We shall evaluate $R(x)$ using both the spectral function approach and the uncorrelated Fermi sea. We have integrated over the momentum up to four times the Fermi momentum for each energy. This gives the normalization of $A$ at the level of $2-3 \%$ which is sufficient for our purposes, but can lead to higher uncertainties in the kinetic energy, which weighs the integral with a higher power of $\vec{p}$. Even a conservative error of $20 \%$ in the kinetic energy has repercussions in the $E M C$ effect only at the level of $2 \%$.

\section{Results and discussion}

\subsection{Nucleonic contribution}

In fig. 7 we show the results for $R(x)$ from the nucleonic contribution calculated as

$$
R_{N}(x)=\frac{F_{2 A, N}\left(x_{A}\right)}{A F_{2 N}(x)}
$$

We do not divide by $F_{2 D}(x)$ as experimentally done. One reason for it is that the techniques used here with the local density approximation cannot be used for deuterium and hence we cannot calculate the nuclei and deuterium 
with the same model. However the price we pay is small. We rely upon the results for $F_{2 D}(x)$ calculated for deuterium in [13. We can see there that for $0<x<0.7 F_{2 D}(x) / F_{2 N}(x)$ ranges between $0.98-1$. Hence, our results should be increased by $1-2 \%$ and we will not worry about this amount. More serious is the region for $x>0.7$, where due to Fermi motion the former ratio increases rapidly. Hence, in that region we should expect to overcount the experiment as it is indeed the case.

In fig. 7 we plot the results obtained for ${ }^{56} \mathrm{Fe}$. This nucleus has $N \neq Z$ but by a little amount. Furthermore, the experimental results are corrected by the isoscalarity factor to convert them into an equivalent isoscalar nucleus [13, 56], hence our calculations done for symmetric nuclear matter are appropriate. We see a minimum around $x=0.6$ as in the experiment and a steep rise at $x>0.75$ as it correponds to Fermi motion [57, 2, 6]. The region around $x=0.6$ agrees well with experiment. However at $x \simeq 0.15-0.2$ we are below the data by about $10-15 \%$. This region will be filled up latter by the mesonic contribution.

In the same figure we show the results obtained with the uncorrelated Fermi sea (local step function distribution) of eq. (72). We observe that $R_{N}(x)$ takes values closer to unity than the results with the spectral function for $x<0.6$ The reduction of $R_{N}(x)$ with the use of the spectral function with respect to a static picture of the nucleus, like Hartree Fock or the equivalent uncorrelated Fermi sea in our case, was already shown and explained in ref. [54. The results here are qualitatively similar to those in [54] and the explanation lies in the increased binding provided by the spectral functions. Both the factor $x / x_{N}$ of eq. (57), as well as the restrictions of phase space $\theta\left(x_{N}\right) \theta\left(1-x_{N}\right)$, are responsible for the decrease of $R_{N}(x)$ in this region.

On the other hand there is a novelty in these results with respect to those in [54]. $R_{N}(x)$ does not go to 1 at $x=0$ as in [54] and are systematically lower in all the range of $x$. The reduction at $x=0$ is easy to see from the formulae. In eq. (55) $F_{2 N}\left(x_{N}=0\right)$ will take a constant value, and with respect to the normalization integral of eq. (21) the novelties in eq. (55) are the extra factors $M / E(\vec{p})$ and $x / x_{N}$ which both go into reducing the contribution of the integrand for off shell nucleons.

The overshooting of the results in the region of $x \simeq 0.8$ was already announced as a result of dividing $F_{2 A}$ by $F_{2 N}$ and not $F_{2 D} / 2$. But the qualitative features due to Fermi motion are reproduced. A very detailed discussion of these effects is given in ref. [6], but qualitatively we can see that

$$
\begin{aligned}
& x_{N} \rightarrow \frac{x_{N}}{x}=\frac{M}{p^{0}-p^{3}} \\
& (x \rightarrow 1)
\end{aligned}
$$

and one can pick up values of $p^{3}$ in the integrations such that $x_{N}<1$. Hence $F_{2 A, N}$ will be different from zero while $F_{2 N}(x=1)=0$, and $R_{N}(x)$ from eq. (73) necessarily goes to $\infty$. 
It is interesting to call on the attention to the crossing of the two lines in fig. 7. The binding effects reduce $R_{N}(x)$. On the other hand Fermi motion increases $R_{N}(x)$ close to 1 , as we noted. Fermi motion is more important in the interacting Fermi sea because now one has larger momentum components. On the other hand the interacting Fermi sea has also more binding. As a consequence we see that at $x<0.6$, where the binding effects dominate over the Fermi motion, $R_{N}(x)$ for the interacting Fermi sea is smaller than with the non interacting Fermi sea, while for $x>0.7$, where the Fermi motion effect dominates, the situation is just opposite.

\subsection{Mesonic contributions}

In figs. 8, 9, 10, 11 we show the mesonic contribution to $R(x)$, together with the nucleonic one discussed above, for different nuclei, ${ }^{6} \mathrm{Li},{ }^{12} \mathrm{C},{ }^{40} \mathrm{Ca}$ and ${ }^{56} \mathrm{Fe}$. The general features are the same in all nuclei, but, of course, the mesonic contribution is smaller in lighter nuclei. The mesonic contribution is calculated with the structure function of ref. [42]. We have also calculated it with the older structure functions of ref. [58, 59]. We find that around $x=0.2$, the results for $R(x)$ decrease in about $3 \%$ if one uses the pion structure function of ref. 58 and increase in about $5 \%$ if one uses the one of ref. [59]. This should give us an idea of the uncertainties of this contribution. In fig. 11, for ${ }^{56} \mathrm{Fe}$, we split the mesonic contribution into the pion and $\rho$-meson ones. We observe, that although the pionic contribution is bigger, the one from the $\rho$-meson cloud is also important, and both of them are positive in all the range of $x$. Similarly, as obtained in other calculations [2, 3, 7] the mesonic contribution vanishes around $x=0.6$ and increases as $x$ decreases. We can see that thanks to the mesonic contribution the agreement with the data becomes much better. The slope of $R(x)$ from $x=0.15$ to $x=0.6$ is not reproduced by the contribution of the nucleons alone, a feature which is shared by the results of ref. [54]. The mesonic contribution comes to produce the right slope.

As we said, the qualitative features of the pionic contribution are similar to those in [2, 3, []. There are also some differences. In ref. [2] the pionic contribution is evaluated assuming different amounts of pion excess in the nucleus, but no evaluation of this excess is made. Furthermore one should recall our warnings in section 4 not to use the excess number in the evaluation. In ref. [3] an actual evaluation is done of the pionic contribution. Even if the formalisms here and there might look quite different, they are actually quite similar and one can see that $\operatorname{Im} D(q) \equiv\left|D(q)^{2}\right| \operatorname{Im} \Pi_{\pi}$ of eq. (48) appears in ref. [3] as $\operatorname{Im} \Pi_{\pi}\left|D_{0}(q)\right|^{2}$, with $|D(q)|^{2}$ changed to $\left|D_{0}(q)\right|^{2}$, which is not very problematic when the pions are off shell. Furthermore, the $\Delta h$ contribution is obtained from an extrapolation of the pionic atom data, which is more problematic when one goes to the off shell situations which one finds here. Furthermore, this $\Delta h$ is taken real in [3], while here the $\Delta h$ Lindhard function is explicitly evaluated as a function of $q^{0}, q$ by keeping the $\Delta$ width. An accurate evaluation of the imaginary parts of the diagrams is necessary 
in order to fulfill the sum rule of eq. (69). On the other hand what one evaluates in ref. [3] is the fractional increase of $R(x)$ with respect to the one in the Sullivan process (deep inelastic scattering with the pion cloud of a free nucleon) [60, 61] and not the absolute value of the contribution of the pionic cloud.

In ref. [7] the absolute contribution of the pion cloud is obtained by subtracting the "free" parts contained in the response function of a free nucleon, as done here. The formalism is similar to ours but some approximations are done which are improved here. For instance the $\Delta h$ contribution is again taken real and other approximations are done to relate some magnitudes to the pion excess number (recall our warnings about this).

The input which we have used for the meson nucleus interaction, $V_{L}(p), V_{T}(p)$, etc, is the one of section 6 . It has the virtue of having been tested in a large variety of reactions and we have not changed it here. This gives us much confidence about the strength of the mesonic contribution obtained here. The fact that it fills up the part of $R(x)$ missed by the nucleonic contribution is certainly a welcome feature which reinforces our confidence on this mesonic model.

The agreement of the results that we obtain with the experimental data can be considered rather good by comparison with results obtained with other theoretical approaches. The trend of the data is well reproduced and the remaining discrepancies are not incompatible with the intrinsic theoretical uncertainties of our model, particularly of the mesonic contribution and more specially of the $\rho$-meson cloud which is somewhat tied to the form factors and the nuclear correlation function, the fact that we divide by $F_{2 N}(x)$ instead of $F_{2 D}(x) / 2$,etc.

Our results for $R(x)$ in ${ }^{40} \mathrm{Ca}$ and ${ }^{56} \mathrm{Fe}$ look already very similar and we have checked that $R(x)$ does not change much for heavier nuclei. Obviously one should take into account that for heavier nuclei $N \neq Z$ and our approach with symmetric nuclear matter should be less accurate. Actual calculations keeping different neutron and proton densities lead to a slight decrease of the minimum of $R(x)$ as $Z$ increases [55].

\section{Conclusions}

We have evaluated the nucleonic and mesonic contributions to the ratio $R(x)$ of the $E M C$ effect, with particular emphasis on an accurate treatment of effects shown in the past to be relevant, like binding effects, Fermi motion and a dynamical (non static) treatment of the nucleons and the mesons in the medium.

In order to avoid having to take some prescription on how to include relativistic effects in the approach, which has led to many discussions in the past, we started with a relativistic approach from the beginning and have made a covariant treatment which allows us to write all magnitudes in terms of nucleon 
and meson propagators in the medium. The approach was made practical by evaluating the relevant magnitudes in an infinite medium and calculating the structure functions in finite nuclei by means of the local density approximation. This procedure is fine for $x>0.1$ but certainly breaks down for $x<0.1$ where there is nuclear shadowing.

We could see that the use of the spectral functions to construct the nucleon selfenergy was relevant in reducing somewhat the ratio $R(x)$ with respect to a static picture of the nucleus, like a shell model, or in our case an uncorrelated Fermi sea. This reconfirmed qualitatively earlier findings in the same direction.

Although our results for the nucleonic contribution differ somewhat from other results in the literature, we share their conclusions that the nucleonic contribution alone does not explain the data, particularly the slope from $x=0$ to $x=0.6$.

On the other hand, we evaluated the contribution from the pion and $\rho$ meson clouds rather accurately. Recent work done before on the contribution of the pion cloud to the $K^{+}$nucleus selfenergy had taught us some important lessons, particularly the importance of using an input which satisfies a sum rule, not trivial to satisfy unless the analytical properties of the pion selfenergy are strictly fulfilled, and the need to avoid any relationship to the "pion excess number". In addition, experience gained in dealing with reactions which involve real and virtual pions allowed us to use information on the pion nucleus selfenergy which is realistic enough and has been tested in many such reactions. Hence, we consider the present calculation of the pionic effects as an improvement over work done in the past and we think these results are rather reliable. However, there are still small uncertainties in the pionic contribution stemming from different results for the pion structure function obtained in different analyses of the Drell-Yan process.

The strength of the pionic effects is moderate. So is the one from the $\rho$-meson cloud, but, when they are added to the nucleonic contribution one obtains a good description of the data in the region of $0.1<x<0.7$.

In summary we could conclude that the main features of the $E M C$ effect can be described in terms of conventional degrees of freedom, nucleons and mesons. It does not exclude explanations in terms of more elementary degrees of freedom like quarks and gluons. It is simply a question of which degrees of freedom are more economical and transparent, as stressed by Jaffe in ref. [15]. The fact that we could deal with these degrees of freedom with a certain accuracy, and establish the relationship of the effects found to familiar concepts in conventional nuclear physics, makes these degrees of freedom rather appropriate to look at the $E M C$ and related effects. 
Acknowledgements

We would like to acknowledge useful discussions with F. Gross, S. Liuti, C. García-Recio, A. Polls and V. Vento.

This work has been partially supported by CICYT contract number AEN 93-1205. One of us E. M. wishes to acknowledge a fellowship from the Ministerio de Educación y Ciencia.

\section{References}

[1] E. M. Collab., J. J. Aubert et al., Phys. Lett. B 123 (1983) 275

[2] C. H. Llewellyn Smith, Phys. Lett. 128 B (1983) 107

[3] M. Ericson and A. W. Thomas, Phys. Lett. 128 B (1983) 112

[4] V. Sanjosé, V. Vento and S. Noguera, Nucl. Phys. A470 (1987) 509; P. González and V. Vento, Mod. Phys. Lett. A8 (1993) 1563

[5] L. L. Frankfurt and M. I. Strikman, Phys. Rep. 160 (1988) 236

[6] R. P. Bickerstaff and A. W. Thomas, J. Phys. G15 (1989) 1523

[7] A. B. Migdal, E. E. Saperstein, M. A. Troitskii and D. N. Voskresenskii, Phys. Rep. 192 (1990) 179

[8] S. V. Akulinichev, S. A. Kulagin and G. M. Vagradov, Pis' ma Zh. Eksp. Teor. Fiz 42 (1985) 105; JETP Lett. 42 (1985) 127; Phys. Lett. 158 B (1985) 485

[9] G. V. Dunne and A. W. Thomas, Phys. Rev. D 33 (1986) 2061

[10] S. V. Akulinichev and S. Shlomo, Phys. Rev. C 33 (1986) 1551

[11] L. L. Frankfurt and M. I. Strikman, Phys. Lett. B 183 (1987) 254

[12] G. L. Li, K. F. Liu and G. E. Brown, Phys. Lett. B 213 (1988) 531

[13] C. Ciofi degli Atti and S. Liuti, Phys. Rev. C 41 (1990) 1100

[14] B. L. Birbrair, E. M. Levin and A. G. Shuvaev, Nucl. Phys. A 491 (1989) 618

[15] R. L. Jaffe, Nucl. Phys. A 478 (1988) 3c

[16] F. Gross and S. Liuti, Phys. Rev. C 45 (1992) 1374

[17] S. Liuti and F. Gross, Phys. Lett. B 356 (1995) 157 
[18] J. Nieves, E. Oset and C. Garcia-Recio, Nucl. Phys. A 554 (1993) 509; ibid, pag. 554

[19] H. C. Chiang, E. Oset and P. Fernández de Córdoba, Nucl. Phys. A 510 (1990) 591

[20] S. K. Singh and E. Oset, Nucl. Phys. A 542 (1992) 587

[21] R. C. Carrasco and E. Oset, Nucl. Phys. A 536 (1992) 445; R. C. Carrasco, E. Oset and L. L. Salcedo, Nucl. Phys. A 541 (1992) 585

[22] S. A. Kulagin, G. Piller and W. Weise, Phys. Rev. C 50 (1994) 1154

[23] N. N. Nikolaev and B. G. Zakharov, Phys. Lett. B 327 (1994) 157

[24] A. L. Fetter and J. D. Walecka, Quantum Field Theory of Many Particle Systems, (McGraw Hill, NY, 1971)

[25] P. Fernández de Córdoba and E. Oset, Nucl. Phys. A 528 (1991) 736

[26] C. Itzykson and J. B. Zuber, Quantum Field Theory (McGraw Hill, NY, 1980)

[27] H. de Vries, C. W. de Jager and C. de Vries, At. Dat. Nucl. Dat. Tables 36 (1987) 495

[28] G. C. Li, I. Sick, R. R. Whitney and M. R. Yearian, Nucl. Phys. A 162 (1971) 583

[29] F. Mandl and G. Shaw, Quantum Field Theory, John Wiley, 1984

[30] E. Amaldi, S. Fubini and G. Furlan, Pion Electroproduction, Springer tracts in modern physics, Vol 83 (Springer, Berlin, 1979)

[31] C. Garcia-Recio, J. Nieves and E. Oset, Phys. Rev. C 51 (1995) 237

[32] M. F. Jiang and D. S. Koltun, Phys. Rev. C 46 (1992) 2462

[33] I. J. R. Aitchison and A. J. G. Hey, Gauge theories in particle physics, Edit Adam Hilger

[34] G. Baym and G. E. Brown, Nucl. Phys. A 247 (1975) 395

[35] W. Y. P. Hwang, J. Speth and G. E. Brown, Z. Phys. A 339 (1991) 383

[36] A. Szczurek and J. Speth, Nucl. Phys. A 555 (1993) 249

[37] L. Heller and A. W. Thomas, Phys. Rev. C 41 (1990) 2756

[38] U. Oelfke, P. U. Sauer and F. Coester, Nucl. Phys A 518 (1990) 593 
[39] W. Melnitchouk, A. W. Schreiber and A. W. Thomas, Phys. Rev. D 49 (1994) 1183

[40] P. Berge et al. Z. Phys. C49 (1991) 187

[41] EMC, J. J. Aubert et al., Phys. Lett B 114 (1982) 291

[42] M. Glück, E. Reya and A. Vogt, Z. Phys. C 53 (1992) 651

[43] R. Machleidt, K. Holinde and Ch. Elster, Phys. Reports 149 (1987) 1

[44] E. Oset and W. Weise, Nucl. Phys. A 319 (1979) 477

[45] E. Oset, P. Fernández de Córdoba, L. L. Salcedo and R. Brockmann, Phys. Reports 188 (1990) 79

[46] P. Fernández de Córdoba and E. Oset, Phys. Rev. C 46 (1992) 1697

[47] S. Fantoni, B. L. Friman and V. R. Pandharipande, Nucl. Phys. A 399 (1983) 51

[48] S. Fantoni and V. R. Pandharipande, Nucl. Phys. A 427 (1984) 473

[49] C. Mahaux, P. F. Bortignon, R. A. Broglia and C. H. Dasso, Phys. Rep. 120 (1985) 1

[50] A. Ramos, A. Polls and W. H. Dickhoff, Nucl. Phys. A 503 (1989) 1

[51] H. Müther, G. Knehr and A. Polls, Phys. Rev. C 52 (1995) 2955

[52] H. Müther, A. Polls and W. H. Dickhoff, Phys. Rev. C 51 (1995) 3040

[53] D. S. Koltun, Phys. Rev. C 9 (1974) 484

[54] C. Ciofi degli Atti and S. Liuti, Phys. Lett. B 225 (1989) 215

[55] C. Ciofi degli Atti and S. Liuti, Phys. Rev. C 44 (1991) R 1269

[56] R. G. Arnold et al., Phys. Rev. Lett. 52 (1984) 727

[57] A. Bodek and J. L. Ritchie, Phys. Rev. D 23 (1981) 1070

[58] J. Badier et al., Z. Phys. C 18 (1983) 281

[59] P. Castorina and A. Donnachie, Z. Phys. C 45 (1990) 497

[60] J. D. Sullivan, Phys. Rev. D 5 (1972) 1732

[61] Ch. H. Chung and W. Y. P. Hwang, Phys. Rev. D 49 (1994) 2221

[62] J. Gómez et al., Phys. Rev. D 49 (1994) 4348

[63] A. C. Benvenuti et al., Phys. Lett. B 189 (1987) 483 
[64] NMC Collaboration, M. Arneodo et al., Nucl. Phys. B 441 (1995) 12

[65] NMC Collaboration, P. Amaudruz et al., Nucl. Phys. B 441 (1995) 3 
$\underline{\text { Figures captions. }}$

- Figure 1: Selfenergy diagrams of the nucleon.

- Figure 2: Electromagnetic form factors for the cases

a) free nucleon, b) Fermi sea with B baryons.

- Figure 3: (a) Feynman diagram for deep inelastic electron-nucleon scattering and (b), electron selfenergy diagram associated.

- Figure 4: Electron selfenergy diagram accounting for electron-pion deep inelastic scattering.

- Figure 5: Diagrams of the electron selfenergy including $1 p h, 1 \Delta h, 1 p h 1 \Delta h$, etc..

- Figure 6: Two diagrams (a) direct and (b) crossed, which contribute to Compton $\gamma \pi$ scattering.

- Figure 7: Results of $R_{N}(x)$ for ${ }^{56} \mathrm{Fe}$. Solid line: using the spectral function; dashed line: using the uncorrelated Fermi sea. Experimental points from ref. [62] (solids dots), ref. [63] (open squares).

- Figure 8: Results of $R(x)$ for ${ }^{6} \mathrm{Li}$. Solid lines: whole calculation including the nucleons and the mesons; dashed line: contribution of the nucleons. Experimental points from ref. [64] (solid dots). Density for ${ }^{6} \mathrm{Li}$ from ref. [28].

- Figure 9: Same as fig. 8 for ${ }^{12} C$. Experimental points from ref. 664 (solid dots), ref. 62 (open squares).

- Figure 10: Same as fig. 8 for ${ }^{40} \mathrm{Ca}$. Experimental points from ref. 65 (solid dots), ref. 62] (open squares).

- Figure 11: Results for $R(x)$ for ${ }^{56} \mathrm{Fe}$. Solid line: whole calculation including the nucleons and the mesons; dashed line: contribution of the nucleons; dot-dashed line: contribution of nucleons plus pions. Experimental points from ref. [62] (solid dots), ref. [63] (open squares). 
Table I

\begin{tabular}{|c|c|c|c|c|c|c|}
\hline & & $<\mathrm{T}>$ & {$[\mathrm{MeV}]$} & $<\mid \mathrm{H}$ & $-\mathrm{M} \mid>[\mathrm{MeV}]$ & $\left|\varepsilon_{A}\right|[\mathrm{MeV}]$ \\
\hline${ }^{6} \mathrm{Li}$ & UFS & 9.3 & & 22.1 & & 5.2 \\
\hline & $\mathrm{SF}$ & 18.8 & & 33.8 & & 5.2 \\
\hline${ }^{12} \mathrm{C}$ & UFS & 13.7 & $(17.0)$ & 31.0 & $(23.0)$ & 8.0 \\
\hline & SF & 31.7 & $(37.0)$ & 50.4 & $(49.0)$ & 7.8 \\
\hline${ }^{40} \mathrm{Ca}$ & UFS & 16.0 & (16.5) & 33.5 & $(26.6)$ & 8.5 \\
\hline & $\mathrm{SF}$ & 40.4 & $(36.0)$ & 58.6 & $(52.1)$ & 8.6 \\
\hline${ }^{56} \mathrm{Fe}$ & UFS & 16.1 & $(17.0)$ & 34.1 & $(25.0)$ & 8.9 \\
\hline & $\mathrm{SF}$ & 40.4 & $(33.0)$ & 58.6 & (49.8) & 8.7 \\
\hline
\end{tabular}

UFS: uncorrelated Fermi sea. SF: spectral function. The numbers in brackets correspond to those obtained in ref. [54] respectively for the Hartree-Fock and spectral functions (the latter called there SRC, from short range correlations). 


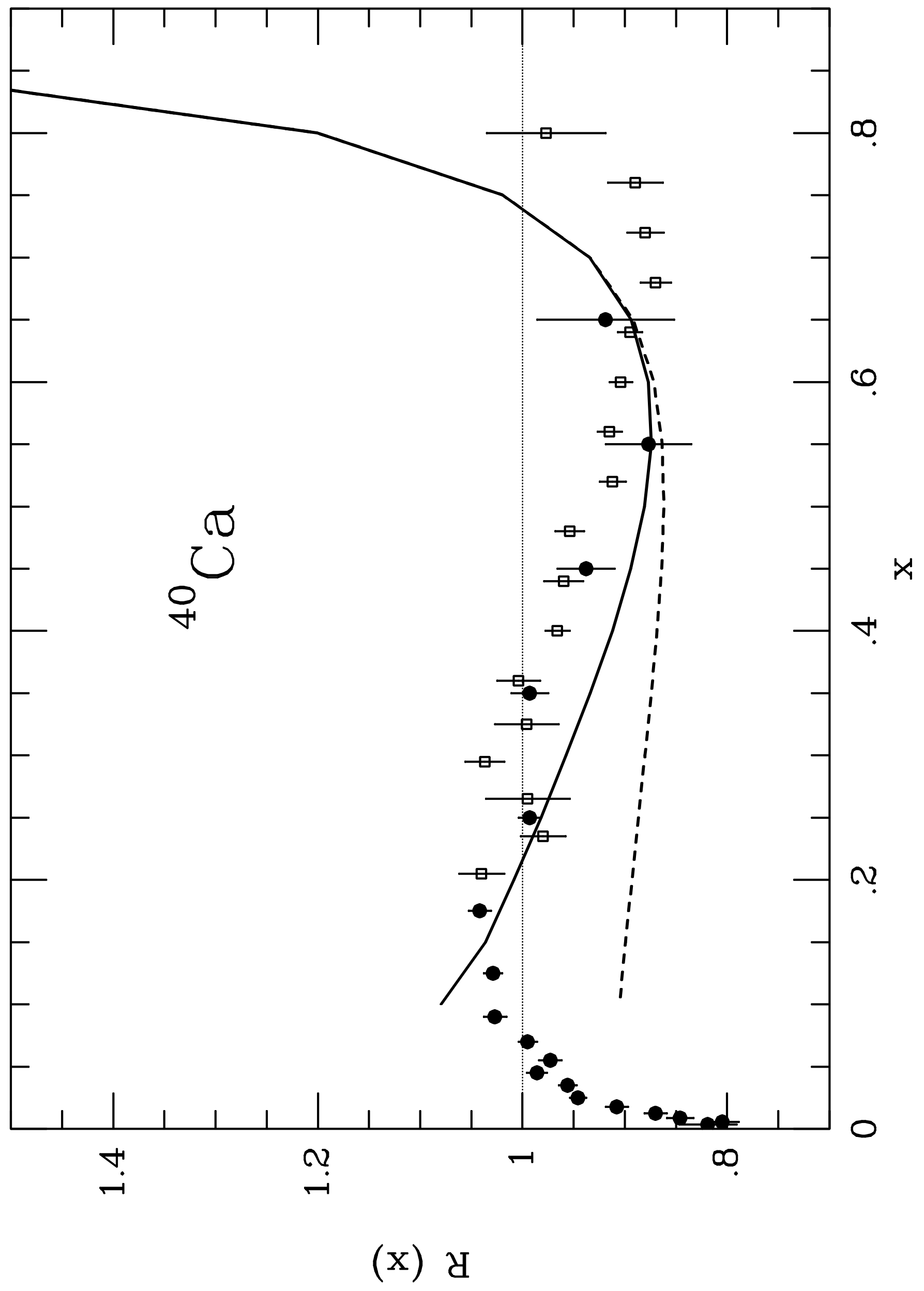




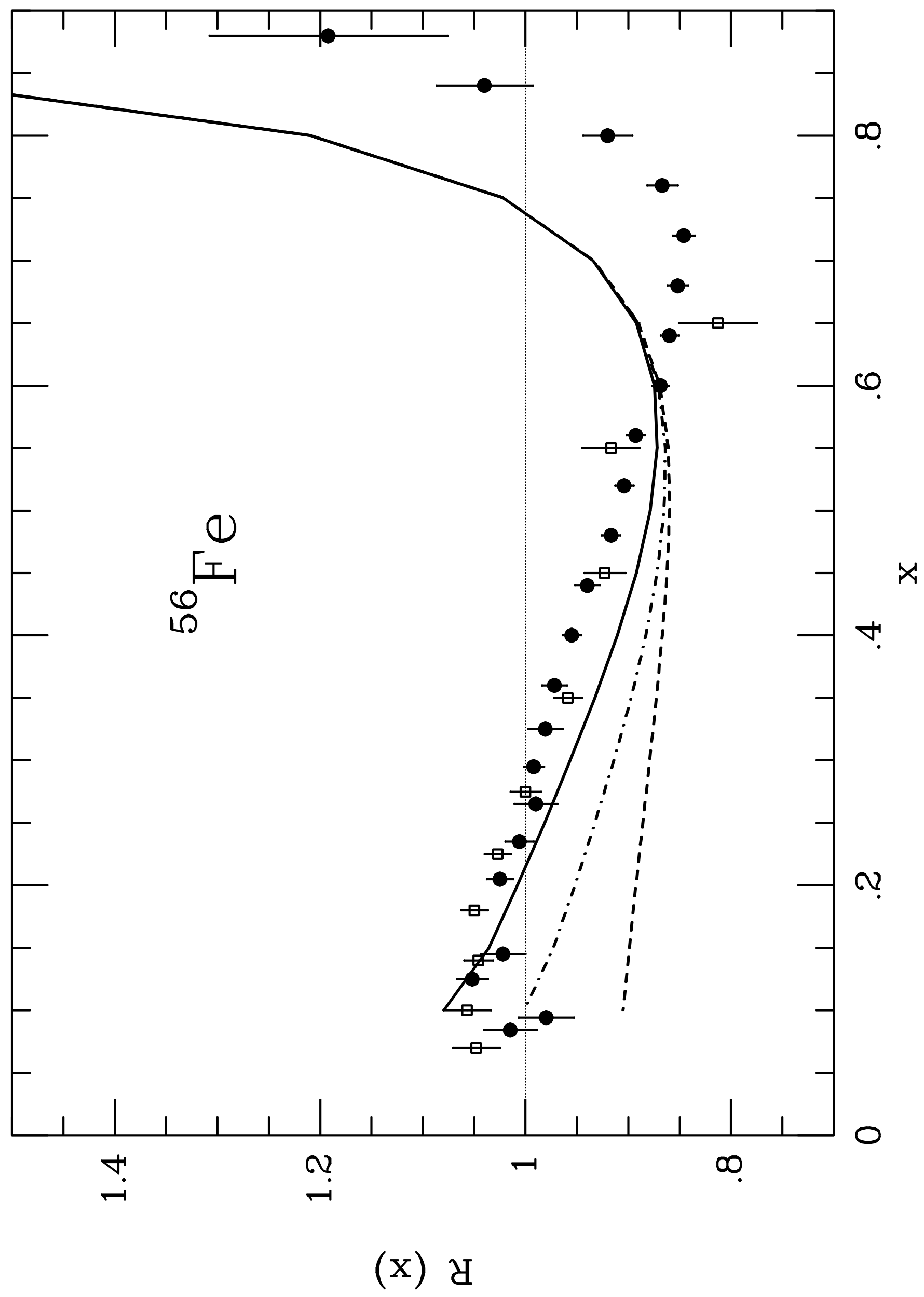




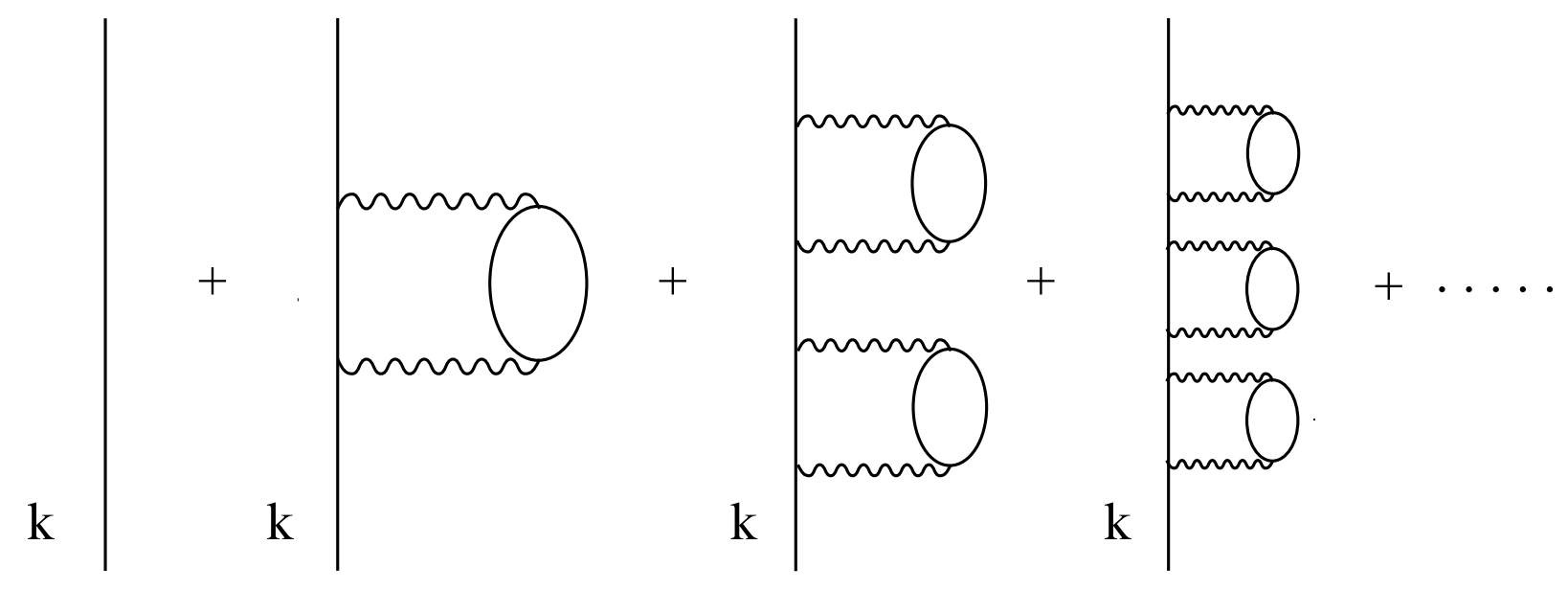

fig. 1 


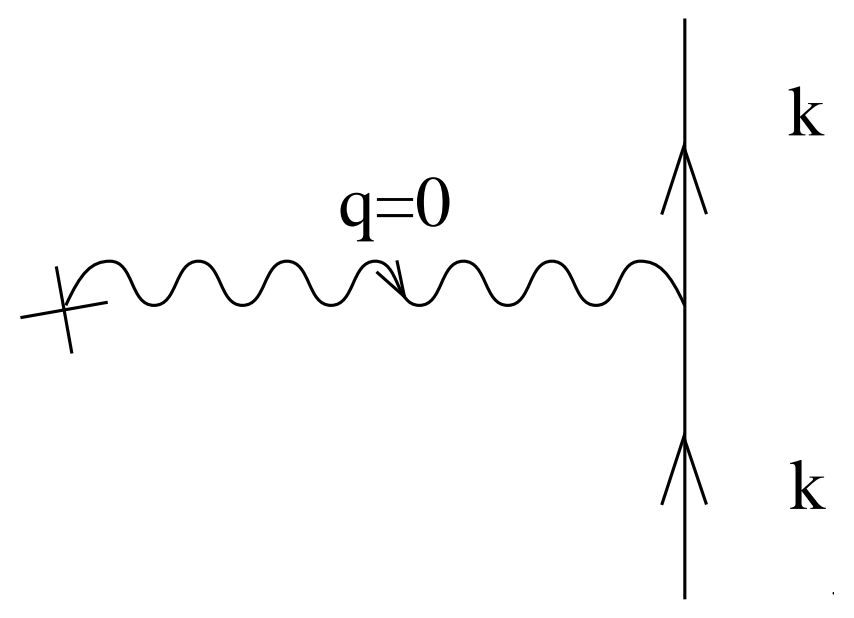

a)

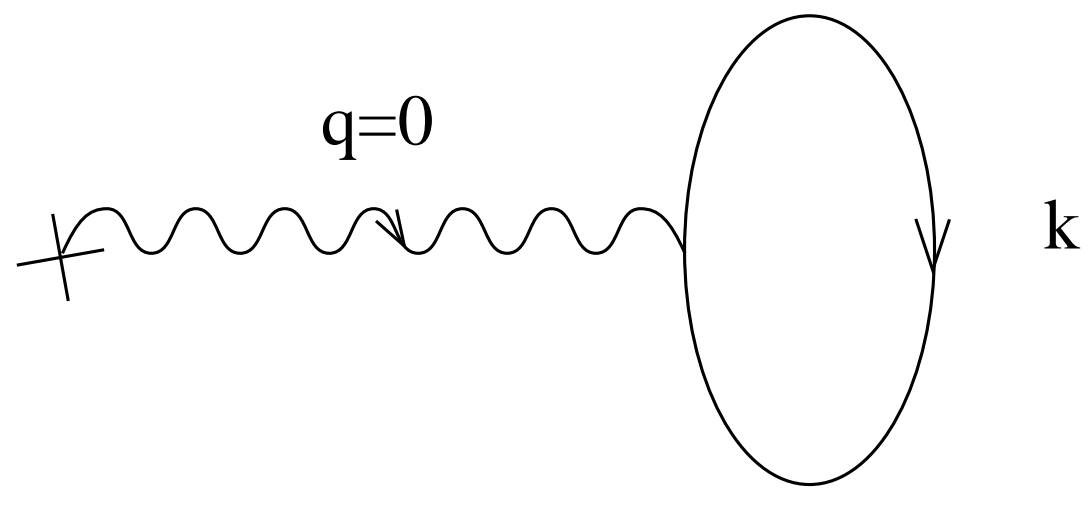

b)

Fig. 2 


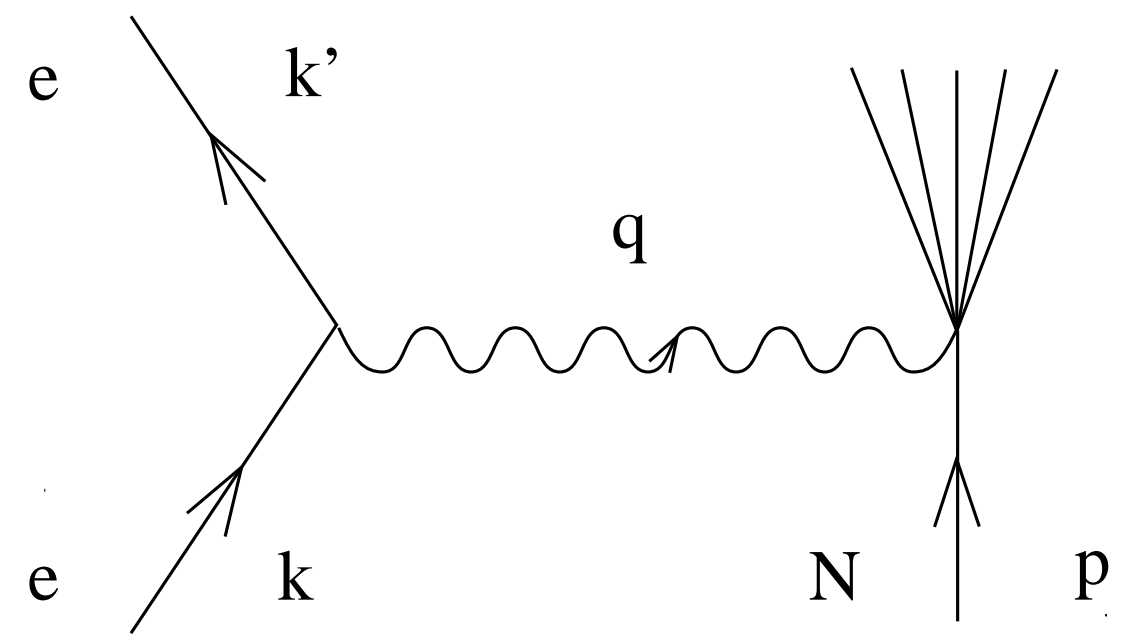

a)

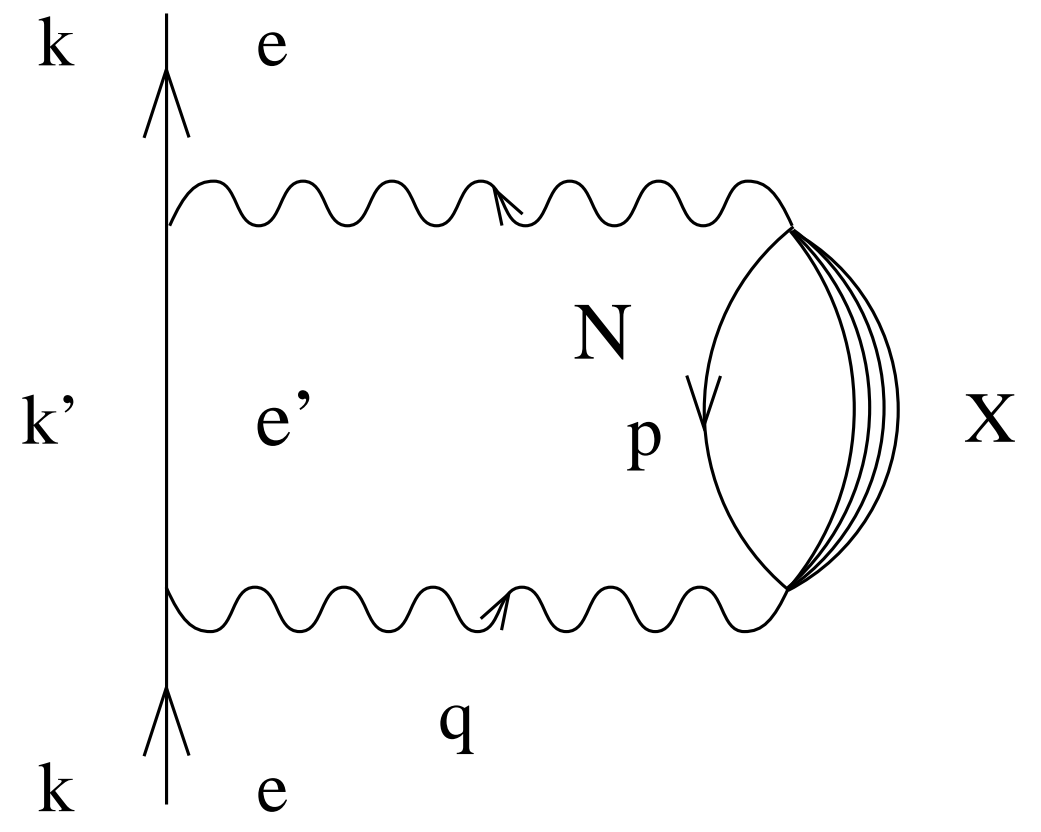

b)

Fig. 3 


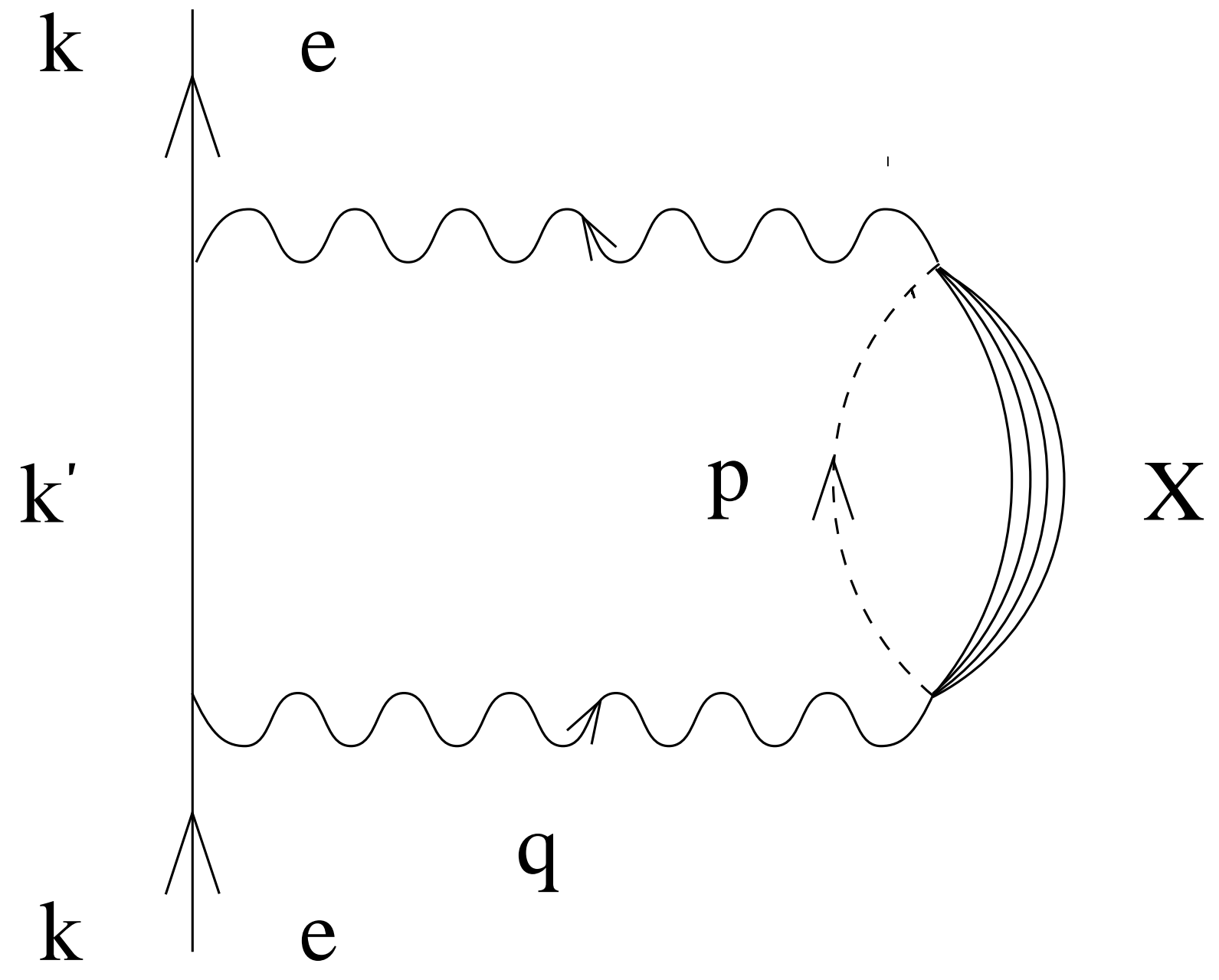

Fig. 4 


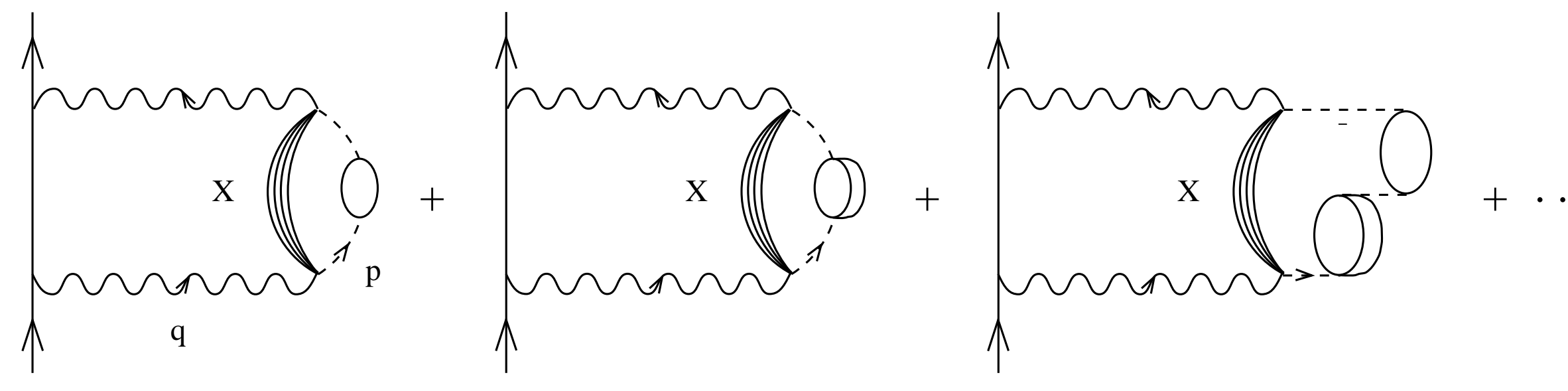

Fig. 5 


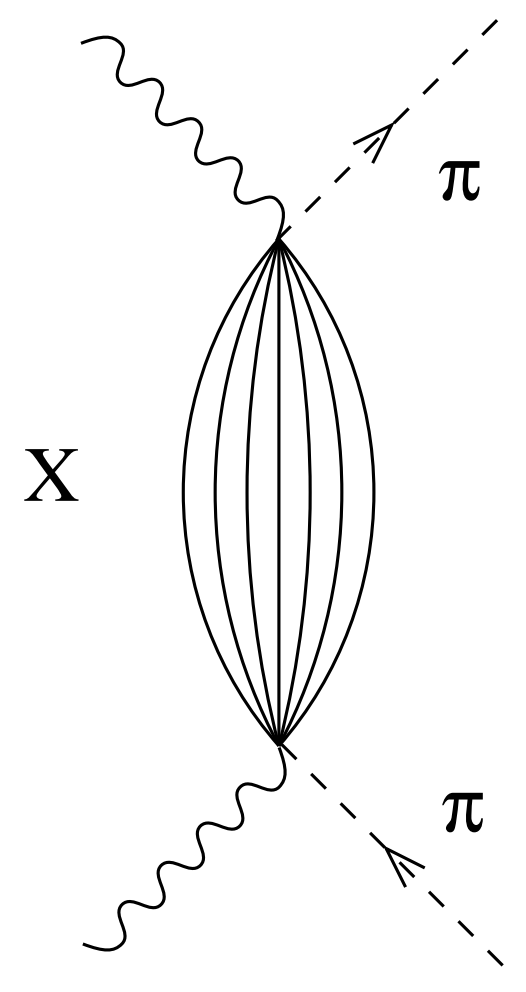

a)

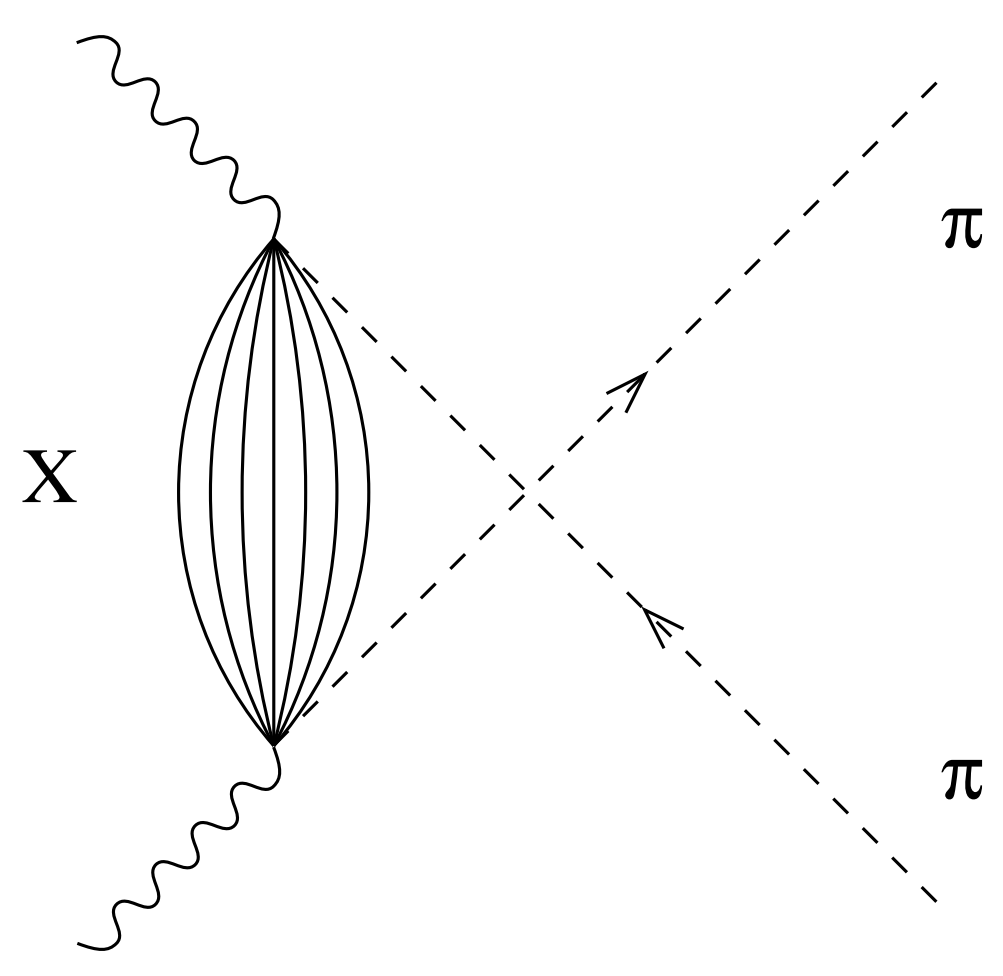

b)

Fig. 6 


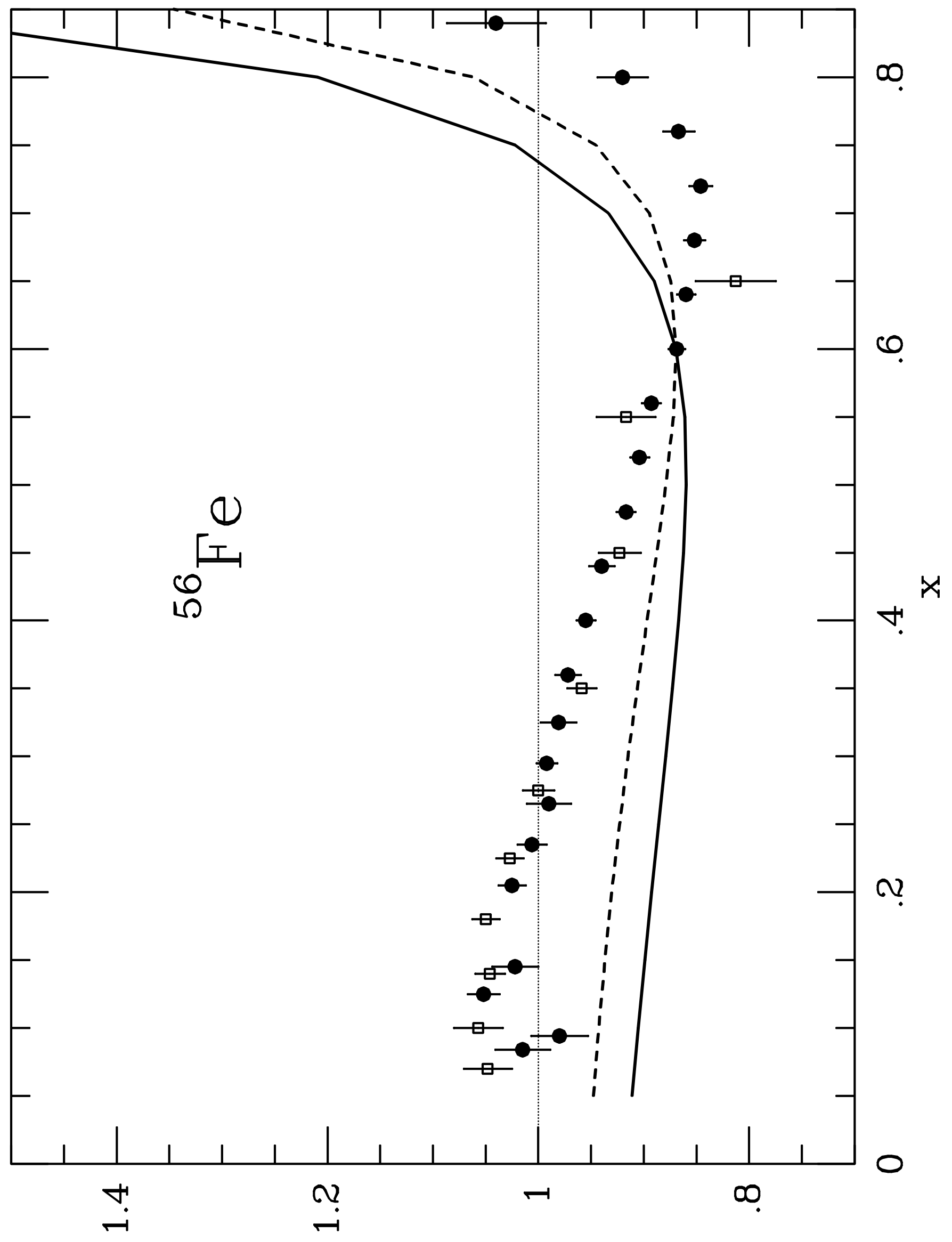

(x) ${ }^{\mathrm{N}} \mathrm{d}$ 


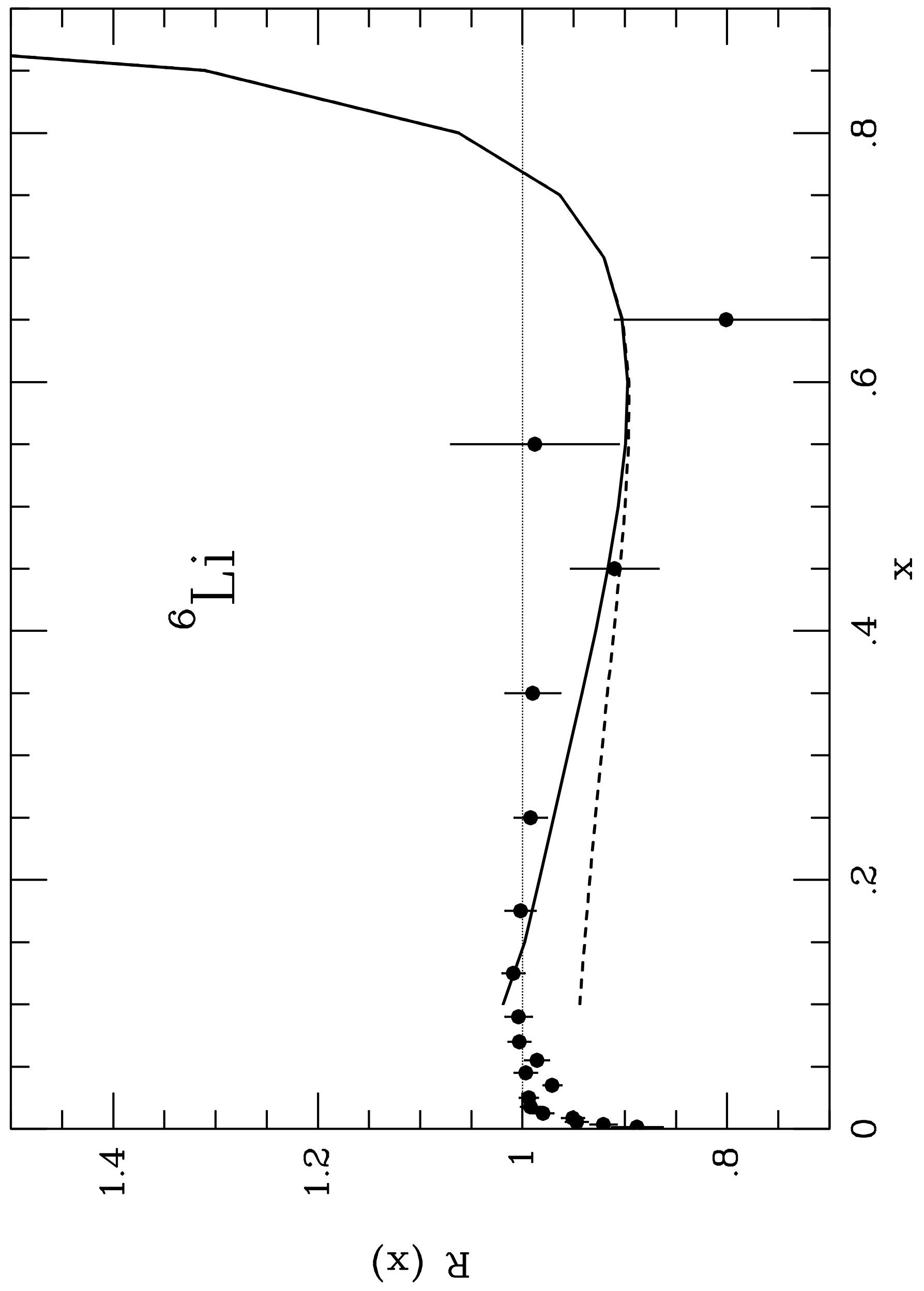




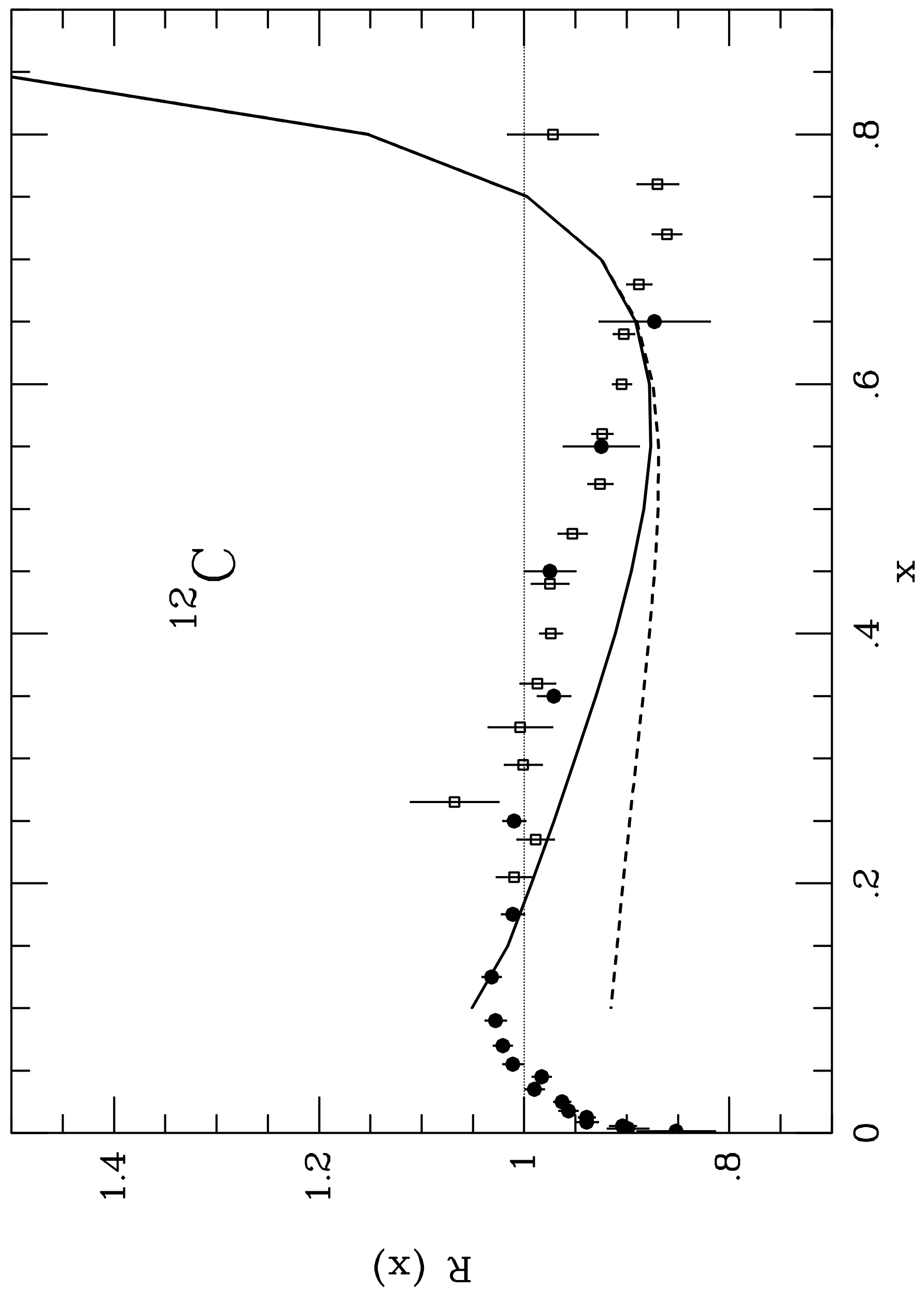

